\title{
A Method for the Computational Modeling of the Physics of Heart Murmurs
}

\author{
Jung Hee Seo ${ }^{1}$, Hani Bakhshaee ${ }^{1}$, Guillaume Garreau ${ }^{2}$, Chi Zhu ${ }^{1}$, Andreas Andreou ${ }^{2}$, William R. Thompson ${ }^{3}$ and \\ Rajat Mittal ${ }^{1, *}$ \\ ${ }^{1}$ Department of Mechanical Engineering, Johns Hopkins University, Baltimore, MD, 21218 \\ ${ }^{2}$ Department of Electrical and Computer Engineering, Johns Hopkins University, Baltimore, MD, 21218 \\ ${ }^{3}$ School of Medicine, Johns Hopkins University, Baltimore, MD, 21218
}

\begin{abstract}
A computational method for direct simulation of the generation and propagation of blood flow induced sounds is proposed. This computational hemoacoustic method is based on the immersed boundary approach and employs high-order finite difference methods to resolve wave propagation and scattering accurately. The current method employs a two-step, one-way coupled approach for the sound generation and its propagation through the tissue. The blood flow is simulated by solving the incompressible Navier-Stokes equations using the sharp-interface immersed boundary method, and the equations corresponding to the generation and propagation of the three-dimensional elastic wave corresponding to the murmur are resolved with a high-order, immersed boundary based, finite-difference methods in the time-domain. The proposed method is applied to a model problem of aortic stenosis murmur and the simulation results are verified and validated by comparing with known solutions as well as experimental measurements. The murmur propagation in a realistic model of a human thorax is also simulated by using the computational method. The roles of hemodynamics and elastic wave propagation on the murmur are discussed based on the simulation results.
\end{abstract}

Keywords: Heart sound; Cardiovascular flow; Immersed boundary method; Hemodynamics; Elastic waves; Systolic murmur.

\section{Introduction}

Blood flows associated with many abnormal cardiovascular conditions generate characteristic sounds called "murmurs" or "bruits", and these sounds can be measured on the skin surface using a stethoscope [1-3]. This technique of "auscultation" $[4,5][6,7]$ has been used for over a hundred years to diagnose cardiovascular diseases, but the physical mechanisms that generate these sounds, as well as the physics of sound transmission through the body, are still not well understood. It has long been accepted that the source of most murmurs are disturbances in blood flow caused by obstruction in the vessels, and there have been many previous studies on the dynamics of flows through stenosed or partially obstructed vessels[8-13]. Several previous studies [3, 14-16] have also shown that the murmur is actually related to the pressure fluctuations on the arterial wall or lumen. A complication with understanding the mechanism of murmur however is that the sound is sensed on the skin surface, and thus, the elastic wave that originates from the blood vessel has to propagate through the various tissues and organs of the

\footnotetext{
*Professor, Corresponding Author (mittal@jhu.edu)
} 
body such as the heart, lungs, ribs, and soft tissues. This propagation induces differential damping, diffraction and dispersion in the waves, and there is currently, very little quantitative understanding of this process.

The availability of ultra-sensitive, low-power, low-cost compact acoustic sensors and sophisticated signal analysis algorithms has rekindled the possibility of developing effective systems for automated cardiac auscultation[17, 18]. Such a diagnostic modality would be non-invasive and inexpensive, and could be highly effective in the screening for particular heart conditions, longitudinal (tracking over time) assessment of cardiac health, 24/7, continuous, at-home health monitoring; and cardiac health assessment in rural and underdeveloped areas where access to specialists is limited. However, automated auscultation systems based purely on empirical knowledge and correlations have had very limited success[6,7]. A better understanding of the physics of murmur generation, propagation and detection could provide the insights needed in order to implement more effective automated auscultation systems, and this is the primary motivation for the current modeling and analysis effort.

It is worth pointing out that experiments are inherently limited in their ability to provide clear physical insights into the relationship between the causal mechanisms and the measured sounds. This is because concurrent measurement of the implicated hemodynamics as well as the measured murmurs is quite difficult in-vivo, and invitro experiments can tackle only very simplified models. Consequently, the entire 200 years old practice of cardiac auscultation with a stethoscope is based primarily on empirical knowledge, and relies on correlations and the significant experiential learning of practicing physicians.

In this context, computational modeling offers a promising modality for exploring the physics of heart murmurs. Previous work on computational modeling of heart murmurs is however, quite limited. In earlier studies [14-16], the propagation of heart murmurs has been modeled with the assumption of a homogeneous medium and that too, for compression (or bulk) wave only. However, biological tissue is a viscoelastic material, and the wave propagation mechanism involves both compression and shear waves, and viscous dissipation as well. Furthermore, in soft tissue, there is a significant scale disparity between the compression and shear waves, with the compression wave speed being more than 100 times faster than the shear wave [19]. This poses a significant challenge when compared to the situation of a pure acoustic wave in a fluid medium.

There have been a few modeling studies employing finite-element [20] or boundary-element based methods [21] on wave propagation in tissue-like materials, but these studies were conducted for highly simplified cases with prescribed sources. In order to more fully understand the relationship between cause (disease) and effect (sound 
measured on the skin surface), the hemodynamics associated with the murmur has to be investigated concurrently, while considering the complete elastic wave dynamics including compression and shear waves propagation, and wave scattering and dissipation. As has been done in computational aeroacoustics (CAA)[22][23] and hydroacoustics fields[24, 25], the direct simulation of blood flow-induced sounds (an approach we refer to as "computational hemoacoustics (CHA)") has the potential to provide an unprecedented understanding of heart murmurs, and this forms the primary motivation for the present study.

In the current paper, we present a computational method for the direct simulation of the generation of blood flow-induced sounds and the propagation of these waves through a tissue-like material. In order to apply this method to the complex geometries associated with biological structures, the method is based on the immersed boundary method (IBM)[26] framework. In the present approach, we employ a two-step, one-way coupled method for the simulation of the hemodynamics and the wave dynamics. Blood flow in the vessel is simulated by solving the incompressible Navier-Stokes equations using the sharp-interface immersed boundary method, and the threedimensional elastic wave generation and propagation in the surrounding viscoelastic tissue-like material is resolved with a high-order finite difference immersed boundary method in the time-domain. The immersed boundary, finite difference method allows us to address complex geometries with ease, and the spatial variation of material properties can be incorporated without difficulty. The time-domain method employed here is also conducive to modeling the broad-band signal that is usually associated with these murmurs.

The paper is organized as follows: In Section 2, the governing equations and the computational method are described in detail. In Section 3, two canonical elastic wave propagation problems are considered for the basic verification of the numerical method. This is followed by a detailed study of the murmur associated with a simplified model of an aortic stenosis. Aortic stenosis is the narrowing of the aortic valve due to a variety of causes including age related calcification, rheumatic fever, and certain infections and congenital heart defects[27]. Significant stenoses can result in hypertrophy (thickening of the heart wall), hypertension, angina (chest pains) and cardiac arrhythmias. Interestingly, while moderate aortic stenoses do not generate these symptoms, they do generate a systolic (during the contraction phase of the heart) murmur that can provide an early warning of those conditions. The increasing incidence of those conditions in an aging population[28] makes aortic stenosis a very appropriate target for the current computational study. The results from the modeling of the aortic stenosis murmurs in a highly simplified configuration are compared with companion experiments in order to provide a strong validation of the 
current method. Results from these hemoacoustic simulations are used for a discussion of the mechanism of blood flow induced sounds. The present computational method is then applied to the simulation of murmur propagation in a realistic, human thorax to demonstrate the capability of the present method. Finally, in section Sec. 4 , we present some concluding remarks.

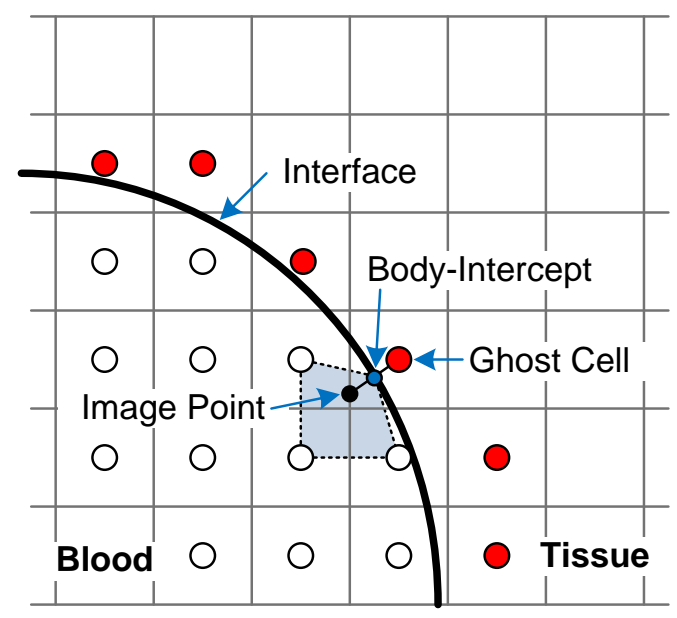

Figure 1: Schematic of the sharp interface immersed boundary method used for the flow solver

\section{Computational Method}

\subsection{Immersed Boundary Flow Solver}

The computational hemoacoustics (CHA) method developed here is based on a two-step, one-way coupled approach where in the first step, the hemodynamic flow field in the blood vessel is simulated by solving the incompressible Navier-Stokes equations. In the second step, the time-varying traction on the vessel wall generated by the flow is used as the source for modeling the wave propagation in the surrounding tissue medium, and this employs a linear elastic wave equation.

For the flow at moderate or high Reynolds numbers in large blood vessels, blood acts as a Newtonian fluid, and thus, the dynamics are governed by the following incompressible Navier-Stokes equations.

$$
\nabla \cdot \vec{U}=0, \quad \frac{\partial \vec{U}}{\partial t}+(\vec{U} \cdot \nabla) \vec{U}+\frac{1}{\rho_{0}} \nabla P=v_{0} \nabla^{2} \vec{U}
$$

where $\vec{U}=(U, V, W)$ is the blood flow velocity, $P$ is the pressure, $\rho_{0}$ and $v_{0}$ are the density and kinematic viscosity of the blood. This equation is solved in the usual way with Dirichlet boundary conditions for velocity and Neumann boundary conditions for pressure. The incompressible Navier-Stokes equations (Eq. 1) are advanced in time using a 
fractional-step based algorithm[29]. A hybrid second-order upwind and central differencing is used for the convection term and a second-order central-scheme is used for the diffusion terms. Time-integration is performed implicitly with the second-order Crank-Nicolson method and the convection term is updated iteratively.

In order to deal with complicated internal boundaries, we employ the sharp-interface immersed boundary method of Mittal et al.[30]. In this method, a surface of the immersed body is represented by an unstructured mesh which consists of triangular elements. At the pre-processing stage, all Cartesian grid cells whose centers are in the flow domain are identified and tagged as "fluid" cells and the other cells outside the flow region are "solid" cells. A solidcell which has at least one fluid-cell neighbor is tagged as a "ghost-cell" (see Fig. 1), and the boundary condition is imposed by specifying an appropriate value at this ghost cell. In the ghost-cell method of Mittal et al.[30] a "normal probe" is extended from the center of a ghost cell to intersect with the immersed boundary (at a body denoted as the "body intercept"). The probe is extended into the fluid domain to the "image point" such that the body-intercept lies midway between the image and the ghost point. A linear interpolation is used along the normal probe to compute the value at the ghost-cell based on the boundary condition. The value at the image-point itself is computed through a tri-linear (in 3D) interpolation from the surrounding fluid cell values. This procedure leads to a nominally secondorder accurate specification of the boundary condition on the immersed boundary. All of the details of this flow solver are presented in Ref.[30], and will therefore not be repeated here. Comprehensive verification of the solver can also be found in Ref.[30] and validation against experimental data for simple as well as complex configurations can be found in Refs.[31-33].

\subsection{Murmur Propagation}

\subsubsection{Governing Equations}

The generation and propagation of elastic waves in the biological tissue surrounding the hemodynamic domain are modeled by the generalized Hooke's law[34] with a Kelvin-Voigt viscoelastic model[35][36]. The governing equations for stress and velocity fluctuations are written as;

$$
\begin{aligned}
& \frac{\partial p_{i j}^{\prime}}{\partial t}+\lambda \frac{\partial u_{k}^{\prime}}{\partial x_{k}} \delta_{i j}+\mu\left(\frac{\partial u_{i}^{\prime}}{\partial x_{j}}+\frac{\partial u_{j}^{\prime}}{\partial x_{i}}\right)=0 \\
& \rho_{s} \frac{\partial u_{i}^{\prime}}{\partial t}+\frac{\partial p_{i j}^{\prime}}{\partial x_{j}}=\eta \frac{\partial}{\partial x_{j}}\left(\frac{\partial u_{i}^{\prime}}{\partial x_{j}}+\frac{\partial u_{j}^{\prime}}{\partial x_{i}}\right)
\end{aligned}
$$


where $p_{i j}^{\prime}$ is the fluctuating elastic stress, $u_{i}^{\prime}$ is the fluctuating velocity, $\rho_{s}$ is the density of the tissue, $\lambda$ and $\mu$ are the first and second Lame's constants, and $\eta$ is the viscosity of the tissue. The above equations describe the propagation of bulk (compression) and shear waves, as well as the dissipation of the waves by viscosity. The speed of the compression wave is based on the bulk modulus, $K=\lambda+2 / 3 \mu$, and given by $c_{b}=\sqrt{K / \rho_{s}}$, while the speed of the shear wave is defined with the shear modulus, $G=\mu$ and $c_{s}=\sqrt{G / \rho_{s}}$.

The boundary conditions on the solid (fixed) wall are as follows:

$$
\frac{\partial p_{i j}^{\prime}}{\partial n}=0, \quad u_{i}^{\prime}=0
$$

and on a traction free boundary (such as the tissue-air interface), the boundary condition is given by;

$$
p_{i j}^{\prime} n_{j}=0, \quad\left[\lambda \frac{\partial u_{k}^{\prime}}{\partial x_{k}} \delta_{i j}+\mu\left(\frac{\partial u_{i}^{\prime}}{\partial x_{j}}+\frac{\partial u_{j}^{\prime}}{\partial x_{i}}\right)\right] n_{j}=0 .
$$

In the present study, we assume that the viscous shear stress induced by the fluid is much smaller than the pressure force, and thus the dominant force at the fluid boundary is the wall normal pressure force. Thus, at the boundary between the blood vessel and the surrounding tissue, the following condition is applied:

$$
p_{i j}^{\prime} n_{j}=P^{\prime} n_{i}, \quad\left[\lambda \frac{\partial u_{k}^{\prime}}{\partial x_{k}} \delta_{i j}+\mu\left(\frac{\partial u_{i}^{\prime}}{\partial x_{j}}+\frac{\partial u_{j}^{\prime}}{\partial x_{i}}\right)\right] n_{j}=-\frac{\partial P^{\prime}}{\partial t} n_{i}
$$

where $n_{j}$ is the surface normal vector, and $P^{\prime}=P-\bar{P}$ is the hydrodynamic pressure fluctuation on the lumen wall. The first condition corresponds to the continuity of normal stress and the second one is derived from the time derivative of the first condition.

The velocity of the blood-tissue interface associated with the elastic waves generated by the hemodynamic pressure can be scaled as $u^{\prime} \sim \mathrm{O}\left(P / \rho_{s} c_{b}\right)[37,38]$ For the tissue-like material, the ratio of this velocity to the hemodynamic velocity scale can be estimated as $u^{\prime} / U \sim \mathrm{O}\left(U / c_{b}\right)$. For the applications considered in the present study, this ratio is about $\mathrm{O}\left(10^{-3}\right)$, and thus the effect of elastic wave on the hemodynamics can be neglected and the hemodynamics and elastic wave propagation can be solved in a sequential, one-way coupled manner. 
We note that, however, in practical situations, especially with pulsatile blood flows, the vessel wall (blood-tissue interface) could deform and introduce additional effects on the elastic wave generation and propagation. These effects are not taken into account in the present model.

\subsubsection{Discretization Methodology}

The numerical method employed for simulating the propagation of the elastic wave in the tissue is one primary contribution of the current paper and the method is described here in detail.

It is noted that the objective here is to accurately simulate the propagation of murmur frequencies ranging up to about $1000 \mathrm{~Hz}$ in the human thorax. For typical shear wave speeds of interest here, the shortest wavelengths to be resolved are therefore of the order of a few millimeters. Given the size of a typical thorax of around $0.3 \mathrm{~m}$, this implies that the shortest wavelengths have to travel distances that exceed 100 times their wavelengths. Given the possibility of reflections, the actual distance of propagation might actually be higher than this. Thus, the numerical method is required to accurately resolve this wave propagation with minimal numerical dispersion and dissipation errors and for this reason, the elastic wave equations, Eq. (2) are solved by a high-order finite difference method. More specifically, the governing equations are discretized in space with a sixth-order central compact finite difference scheme[39] and integrated in time using a four-stage Runge-Kutta (RK4) method[40]. The first derivative is evaluated with the sixth-order compact scheme as follows

$$
\alpha f^{\prime}{ }_{i-1}+f^{\prime}{ }_{i}+\alpha f^{\prime}{ }_{i+1}=a \frac{f_{i+1}-f_{i-1}}{2 h}+b \frac{f_{i+2}-f_{i-2}}{4 h},
$$

where $\alpha=1 / 3, a=14 / 9$, and $b=1 / 9$. Near the immersed boundaries and domain boundaries, the following boundary schemes are used.

$$
\begin{gathered}
f^{\prime}{ }_{i-1}+4 f^{\prime}{ }_{i}+f^{\prime}{ }_{i+1}=3\left(f_{i+1}-f_{i-1}\right) / h, \\
2 f^{\prime}{ }_{i}+4 f^{\prime}{ }_{i+1}=\left(-5 f_{i}+4 f_{i+1}+f_{i+2}\right) / h .
\end{gathered}
$$

Equation (7) is fourth-order and (8) is third-order accurate. These boundary schemes are discussed and suggested in Ref.[39]. Since the central compact scheme has no numerical dissipation, an implicit spatial filtering proposed by Gaitonde et al.[41] is applied to suppress aliasing errors and ensure numerical stability. A general $M$-th order spatial filtering formulation is written as 


$$
\alpha_{f} \tilde{f}_{i-1}+\tilde{f}_{i}+\alpha_{f} \tilde{f}_{i+1}=\sum_{n=0}^{M / 2} \frac{a_{n}}{2}\left(f_{i-n}+f_{i+n}\right)
$$

where $-0.5<\alpha_{f}<0.5$ is a free parameter, and coefficients $a_{n}$ can be found in Ref.[41]. For a value of $\alpha_{f}$ which is close to 0.5 , the filtering truncates only very high wave number components. In this study, we applied an 8th-order filtering with $\alpha_{f}=0.47$ in the interior region. Near the boundaries, reduced, (8th, 6th, 4 th, and $2^{\text {nd }}$ ) order filtering is used.

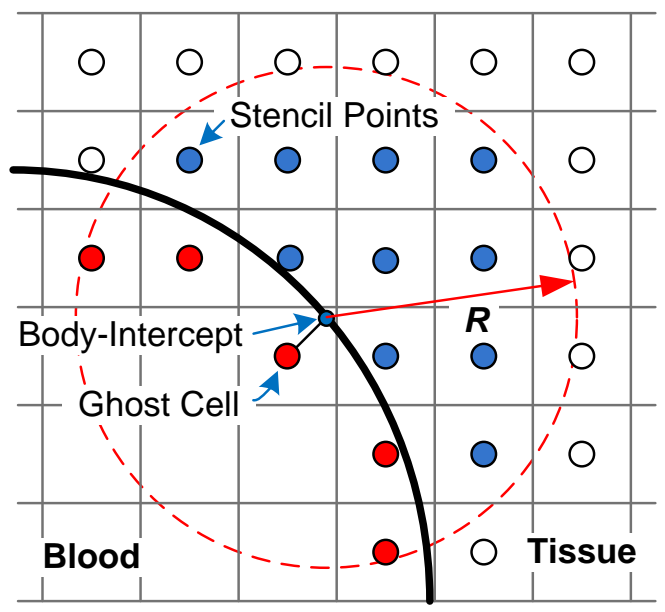

Figure 2: Schematic of the Approximating Polynomial Method (APM) used for the elastic wave solver

\subsubsection{Immersed Boundary Formulation}

One difficulty in solving the structural dynamics or the elastic wave equations (Eq. 2) using the finite difference method is the application of a traction boundary condition (Eqs. $4 \& 5$ ). To apply the traction boundary condition, not only the surface normal but also surface tangential gradient needs to be imposed on the same cell. Applying the tangential gradient with a finite difference approximation is however not trivial, especially for the immersed boundary method. This issue is previously discussed by Luo et al.[42], and they proposed the use of a least-squares polynomial fit to apply the traction boundary condition in the immersed boundary method. In our previous study, we adopted this approach and proposed an approximating polynomial method (APM)[43] for the higher order treatment of immersed boundaries, and the method was successfully applied to the simulations of acoustic waves using a highorder compact finite difference schemes. In the present study, a similar immersed boundary treatment is employed for solving the elastic wave equations on the Cartesian grid. 
In the current approach, the value at the ghost cell is determined by satisfying the boundary condition at the body-intercept point using polynomials of arbitrary order. Specifically, a generic variable $\phi$ is approximated in the vicinity of the body-intercept point $\left(x_{B I}, y_{B I}, z_{B I}\right)$ in terms of a $N^{\text {th }}$-degree polynomial $\Phi$ as follows:

$$
\phi\left(x^{\prime}, y^{\prime}, z^{\prime}\right) \approx \Phi\left(x^{\prime}, y^{\prime}, z^{\prime}\right)=\sum_{i=0}^{N} \sum_{j=0}^{N} \sum_{k=0}^{N} c_{i j k}\left(x^{\prime}\right)^{i}\left(y^{\prime}\right)^{j}\left(z^{\prime}\right)^{k}, \quad i+j+k \leq N
$$

where $x^{\prime}=x-x_{B I}, y^{\prime}=y-y_{B I}, z^{\prime}=z-z_{B I}$ and $c_{i j k}$ are unknown coefficients. In order to determine these coefficients, known variable values on the stencil points around the body-intercept point (in the region of radius $R$, see Fig. 2) are used. With $M$ data points, the coefficients $c_{i j k}$ can be determined by minimizing the weighted error estimated as:

$$
\varepsilon=\sum_{m=1}^{M} w_{m}^{2}\left[\Phi\left(x_{m}^{\prime}, y_{m}^{\prime}, z_{m}^{\prime}\right)-\phi\left(x_{m}^{\prime}, y_{m}^{\prime}, z_{m}^{\prime}\right)\right]^{2}
$$

where $\left(x_{m}^{\prime}, y_{m}^{\prime}, z_{m}^{\prime}\right)$ is the $m$-th stencil point, and $w_{m}$ is a weight function. In this study, we have found that the following hyperbolic secant weight function is a particularly appropriate and robust choice for the traction boundary condition:

$$
w_{m}=\operatorname{sech}\left(\frac{d_{m}}{l_{\Delta}}\right),
$$

where $d_{m}=\sqrt{x_{m}^{\prime}{ }^{2}+y^{\prime}{ }_{m}{ }^{2}+z_{m}^{\prime}{ }^{2}} \leq R$ is the distance between the $m$-th stencil point and the body intercept point, and $l_{\Delta}$ is a length scale associated with the grid spacing around the body-intercept point. The first point $(m=1)$ is the ghost cell point, and (M-1) stencil points are found in the domain (see Fig. 2). The exact solution of the least-square problem, Eq. (11) is given by

$$
\mathbf{c}=(\mathbf{W V})^{+} \mathbf{W} \phi
$$

where superscript + denotes the pseudo-inverse of a matrix, vector $\mathbf{c}$ and $\phi$ contain coefficients $c_{i j k}$ and the data $\phi\left(x_{m}^{\prime}, y_{m}^{\prime}, z_{m}^{\prime}\right)$ respectively, and $\mathbf{W}$ and $\mathbf{V}$ are the weight and Vandermonde matrices given by 


$$
\mathbf{W}=\left[\begin{array}{ccccccccc}
w_{1} & & & \\
& w_{2} & & \\
& & \ddots & \\
& & & w_{M}
\end{array}\right], \quad \mathbf{V}=\left[\begin{array}{cccccccc}
1 & x_{1}^{\prime} & y_{1}^{\prime} & z_{1}^{\prime} & x_{1}^{\prime 2} & y_{1}^{\prime 2} & z_{1}^{\prime 2} & \ldots \\
& & & \vdots & & & & \\
1 & x_{m}^{\prime} & y_{m}^{\prime} & z_{m}^{\prime} & x_{m}^{\prime}{ }^{2} & y_{m}^{\prime 2} & z_{m}^{\prime}{ }^{2} & \ldots \\
& & & \vdots & & & & \\
1 & x_{M}^{\prime} & y_{M}^{\prime} & z_{M}^{\prime} & x_{M}^{\prime}{ }^{2} & y_{M}^{\prime}{ }^{2} & z_{M}^{\prime}{ }^{2} & \ldots
\end{array}\right] .
$$

Note that $\left(x_{1}^{\prime}, y_{1}^{\prime}, z^{\prime}{ }_{1}\right)$ is the ghost-point and $\mathbf{V}$ is a $M \times L$ matrix where $L$ is the number of coefficients. The pseudoinverse matrix is computed by singular value decomposition (SVD) method. After solving Eq. (13), the coefficients $c_{i j k}$ can be written as a linear combination of $\phi\left(x_{m}^{\prime}, y_{m}^{\prime}, z_{m}^{\prime}\right)$. For example,

$$
\begin{aligned}
& c_{000}=\sum_{m=1}^{M} A(1, m) \cdot \phi\left(x_{m}^{\prime}, y_{m}^{\prime}, z_{m}^{\prime}\right) \\
& c_{100}=\sum_{m=1}^{M} A(2, m) \cdot \phi\left(x_{m}^{\prime}, y_{m}^{\prime}, z_{m}^{\prime}\right), \\
& \vdots
\end{aligned}
$$

where $A(l, m)$ is the $l$-th row, $m$-th column component of matrix $\mathbf{A}=(\mathbf{W V})^{+} \mathbf{W}$, which has dimension of $L \times M$. Additional details about the approximating polynomial method can be found in Ref.[43].

One advantage of this treatment is that various boundary conditions including the traction boundary condition (Eqs. 4 \& 5) can be applied relatively easily within the immersed boundary framework[42], because coefficients $c_{i j k}$ represent the value and derivatives at the body-intercept point $\left(x_{B I}, y_{B I}, z_{B I}\right)$, i.e. :

$$
c_{000}=\phi\left(x_{B I}, y_{B I}, z_{B I}\right), \quad c_{100}=\frac{\partial \phi}{\partial x}\left(x_{B I}, y_{B I}, z_{B I}\right), c_{010}=\frac{\partial \phi}{\partial y}\left(x_{B I}, y_{B I}, z_{B I}\right), \cdots
$$

Therefore, for the various types of boundary conditions, the value at the ghost point can be evaluated using Eqs. (15) $\&$ (16). For the Dirichlet boundary condition at the wall, $\phi\left(x_{B I}, y_{B I}, z_{B I}\right)=\phi_{w}$, the ghost-point value is given by

$$
\phi_{g p}=\left[\phi_{w}-\sum_{2}^{M} A(1, m) \cdot \phi\left(x_{m}^{\prime}, y_{m}^{\prime}, z_{m}^{\prime}\right)\right] / A(1,1),
$$

For the Neumann type boundary condition of a wall normal derivative, $\partial \phi / \partial n\left(x_{B I}, y_{B I}, z_{B I}\right)=\phi^{\prime}{ }_{w}$, the value at the ghost point is given by 


$$
\phi_{g p}=\frac{\phi_{{ }_{w}}^{\prime}-\sum_{2}^{M} \hat{n} \cdot(A(2, m) \hat{i}+A(3, m) \hat{j}+A(4, m) \hat{k}) \cdot \phi\left(x_{m}^{\prime}, y_{m}^{\prime}, z_{m}^{\prime}\right)}{\hat{n} \cdot(A(2,1) \hat{i}+A(3,1) \hat{j}+A(4,1) \hat{k})},
$$

where $\hat{n}$ is the unit wall normal vector at the body intercept point. For the traction boundary condition, the velocity components at the ghost point need to be determined by solving Eq. (4) or (5). In the APM, this can be done by solving the following 3 by 3 matrix equation.

$$
\mathbf{C}_{1}\left[\begin{array}{c}
u_{g p}^{\prime} \\
v_{g p}^{\prime} \\
w_{g p}^{\prime}
\end{array}\right]=\mathbf{T}-\sum_{m=2}^{M}\left\{\mathbf{C}_{m}\left[\begin{array}{c}
u^{\prime}\left(x_{m}^{\prime}, y_{m}^{\prime}, z_{m}^{\prime}\right) \\
v^{\prime}\left(x_{m}^{\prime}, y_{m}^{\prime}, z_{m}^{\prime}\right) \\
w^{\prime}\left(x_{m}^{\prime}, y_{m}^{\prime}, z_{m}^{\prime}\right)
\end{array}\right]\right\},
$$

where $\mathbf{C}_{m}$ is a 3 by 3 matrix:

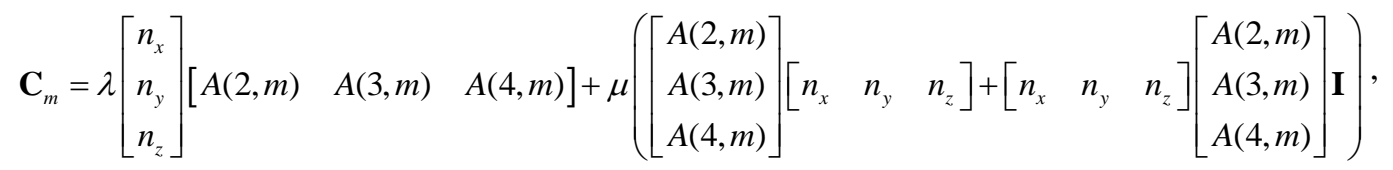

and $\mathbf{T}$ is a traction vector and at the interface, which is given by

$$
\mathbf{T}=\left[\begin{array}{ccc}
-\frac{\partial P^{\prime}}{\partial t} n_{x} & -\frac{\partial P^{\prime}}{\partial t} n_{y} & -\frac{\partial P^{\prime}}{\partial t} n_{z}
\end{array}\right]^{\mathrm{T}}
$$

and $\mathbf{T}=0$ at the traction free boundary.

\subsubsection{Solution procedure}

The governing equation for elastic wave propagation, Eq. (2), is written in the vector form as;

$$
\frac{\partial \vec{Q}}{\partial t}=-\frac{\partial \vec{E}}{\partial x}-\frac{\partial \vec{F}}{\partial y}-\frac{\partial \vec{G}}{\partial z}
$$

where

$$
\vec{Q}=\left[\begin{array}{lllllllll}
p_{x x}^{\prime} & p_{y y}^{\prime} & p_{z z}^{\prime} & p_{x y}^{\prime} & p_{y z}^{\prime} & p_{x z}^{\prime} & u^{\prime} & v^{\prime} & w^{\prime}
\end{array}\right]^{T},
$$




$$
\vec{E}=\left[\begin{array}{l}
(\lambda+2 \mu) u^{\prime} \\
\lambda u^{\prime} \\
\lambda u^{\prime} \\
\mu v^{\prime} \\
0 \\
\mu w^{\prime} \\
\frac{p_{x x}^{\prime}}{\rho_{s}}-\frac{2 \eta}{\rho_{s}}\left(\frac{\partial u^{\prime}}{\partial x}\right) \\
\frac{p_{x y}^{\prime}}{\rho_{s}}-\frac{\eta}{\rho_{s}}\left(\frac{\partial u^{\prime}}{\partial y}+\frac{\partial v^{\prime}}{\partial x}\right) \\
\frac{p_{x z}^{\prime}}{\rho_{s}}-\frac{\eta}{\rho_{s}}\left(\frac{\partial u^{\prime}}{\partial z}+\frac{\partial w^{\prime}}{\partial x}\right)
\end{array}\right], \vec{F}=\left[\begin{array}{l}
\lambda v^{\prime} \\
(\lambda+2 \mu) v^{\prime} \\
\lambda v^{\prime} \\
\mu u^{\prime} \\
\mu w^{\prime} \\
0 \\
\frac{p_{x y}^{\prime}}{\rho_{s}}-\frac{\eta}{\rho_{s}}\left(\frac{\partial u^{\prime}}{\partial y}+\frac{\partial v^{\prime}}{\partial x}\right) \\
\frac{p_{y y}^{\prime}}{\rho_{s}}-\frac{2 \eta}{\rho_{s}}\left(\frac{\partial v^{\prime}}{\partial y}\right) \\
\frac{p_{y z}^{\prime}}{\rho_{s}}-\frac{\eta}{\rho_{s}}\left(\frac{\partial w^{\prime}}{\partial y}+\frac{\partial v^{\prime}}{\partial z}\right)
\end{array}\right], \vec{G}=\left[\begin{array}{l}
\lambda w^{\prime} \\
\lambda w^{\prime} \\
(\lambda+2 \mu) w^{\prime} \\
0 \\
\mu v^{\prime} \\
\mu u^{\prime} \\
\frac{p_{x z}^{\prime}}{\rho_{s}}-\frac{\eta}{\rho_{s}}\left(\frac{\partial u^{\prime}}{\partial z}+\frac{\partial w^{\prime}}{\partial x}\right) \\
\frac{p_{y z}^{\prime}}{\rho_{s}}-\frac{\eta}{\rho_{s}}\left(\frac{\partial w^{\prime}}{\partial y}+\frac{\partial v^{\prime}}{\partial z}\right) \\
\frac{p_{z z}^{\prime}}{\rho_{s}}-\frac{2 \eta}{\rho_{s}}\left(\frac{\partial w^{\prime}}{\partial z}\right)
\end{array}\right] .
$$

All the spatial derivatives on the right-hand side of Eq. (22) are estimated by the compact finite difference scheme, Eqs. (6-8), and the Eq. (22) is integrated in time using a four-stage Runge-Kutta method. The time step size, $\Delta t$, is chosen based on the CFL condition:

$$
\Delta t \leq\left(\max \left(c_{b}, c_{s}\right) \sqrt{\frac{1}{\Delta x^{2}}+\frac{1}{\Delta y^{2}}+\frac{1}{\Delta z^{2}}}\right)^{-1},
$$

At the end of each Runge-Kutta sub-steps, the boundary conditions are imposed by updating the ghost-cell values using Eqs. (17-19). After the final Runge-Kutta step, the spatial filtering, Eq. (9) is applied to suppress high wave number numerical errors.

\section{Result and Discussion}

\subsection{Verification for Elastic Wave Generated by a Point Force}

In order to test the accuracy of current numerical methods on resolving elastic wave generation and propagation in a soft tissue material, we considered a simple benchmark problem of which analytical solution can be found at ease. A test case is an elastic wave generation by a single point force in an unbounded domain. A time harmonic point force in the vertical $(y)$ direction is applied at the origin, and the three-dimensional elastic wave generation and

propagation are computed by Eq. (2) with a body force vector, $\vec{f}=\left[\begin{array}{lll}0 & \sin \left(\omega_{f} t\right) & 0\end{array}\right]^{\mathrm{T}}$ on the right hand side of the second equation. For the material properties, we use density $\rho_{s}=1$, bulk modulus $K=1$, and shear modulus $G=0.01$, because the shear modulus is much smaller than the bulk modulus in soft tissue materials. These material properties 
result in a compression wave speed, $c_{b}=1$, and shear wave speed, $c_{s}=0.1$. The viscosity $\eta$ is set to 0 in this test problem to clearly identify the numerical errors on the wave propagation.

An analytical solution of this problem can be found by using the free space Green's tensor[44] which corresponds to the frequency domain solution of Eq. (2) with zero viscosity and the point force given as above in an infinite space with no outer confinement;

$$
\begin{aligned}
G_{i j}(\vec{r}, \omega)= & \frac{i k_{p}}{12 \pi(\lambda+2 \mu)}\left(\delta_{i j} h_{0}^{(1)}\left(k_{p} r\right)+\left(\delta_{i j}-3 \frac{x_{i} x_{j}}{r^{2}}\right) h_{2}^{(1)}\left(k_{p} r\right)\right), \\
& -\frac{i k_{s}}{12 \pi \mu}\left(-2 \delta_{i j} h_{0}^{(1)}\left(k_{s} r\right)+\left(\delta_{i j}-3 \frac{x_{i} x_{j}}{r^{2}}\right) h_{2}^{(1)}\left(k_{s} r\right)\right)
\end{aligned}
$$

where $k_{p}=\omega / \sqrt{(\lambda+2 \mu) / \rho}$ is a longitudinal wave number, $k_{s}=\omega / \sqrt{\mu / \rho}$ is a shear wave number, $h_{n}^{(1)}$ is the spherical Hankel function of the first kind, $x_{j}$ is the vector from the source to the observer, and $r$ is the magnitude of the vector $x_{j}$. The velocity fluctuation by the point force in the frequency domain is given by

$$
\hat{u}_{i}^{\prime}(\vec{r}, \omega)=-i \omega \cdot G_{i j}(\vec{r}, \omega) \hat{f}_{j}(\omega)
$$

where $\hat{u}_{i}^{\prime}(\omega)=\int u_{i}^{\prime}(t) e^{i \omega t} d t$ and $\hat{f}_{i}(\omega)=\int f_{i}(t) e^{i \omega t} d t$. For the time harmonic, unit point force with the angular frequency, $\omega_{f}$, in the $y$-direction, the resulting velocity fluctuation is given by

$$
u_{i}^{\prime}(\vec{r}, t)=-\omega_{f} \cdot G_{i 2}\left(\vec{r}, \omega_{f}\right) e^{-i \omega_{f} t}
$$

For the numerical solution, a computational domain is set to $-8 \leq(x, y, z) \leq 8$, and this is resolved by $400 \times 400 \times 400$ grid points with a uniform grid spacing of 0.04 in order to test low to high frequency waves, which requires large domain size with small grid spacing. The ETA (Energy Transfer \& Annihilating) non-reflecting boundary condition[45] is applied at all domain boundaries, and 20 grid points are used for a buffer zone at each boundary. The computations are performed for various frequencies, $\omega_{f}=0.2 \pi, 0.4 \pi, 0.6 \pi$, and $0.8 \pi$, and the corresponding compression wave lengths are $\lambda_{b}=10,5,10 / 3,2.5$, and the shear wave lengths are $\lambda s=1,0.5,1 / 3,0.25$, respectively. 

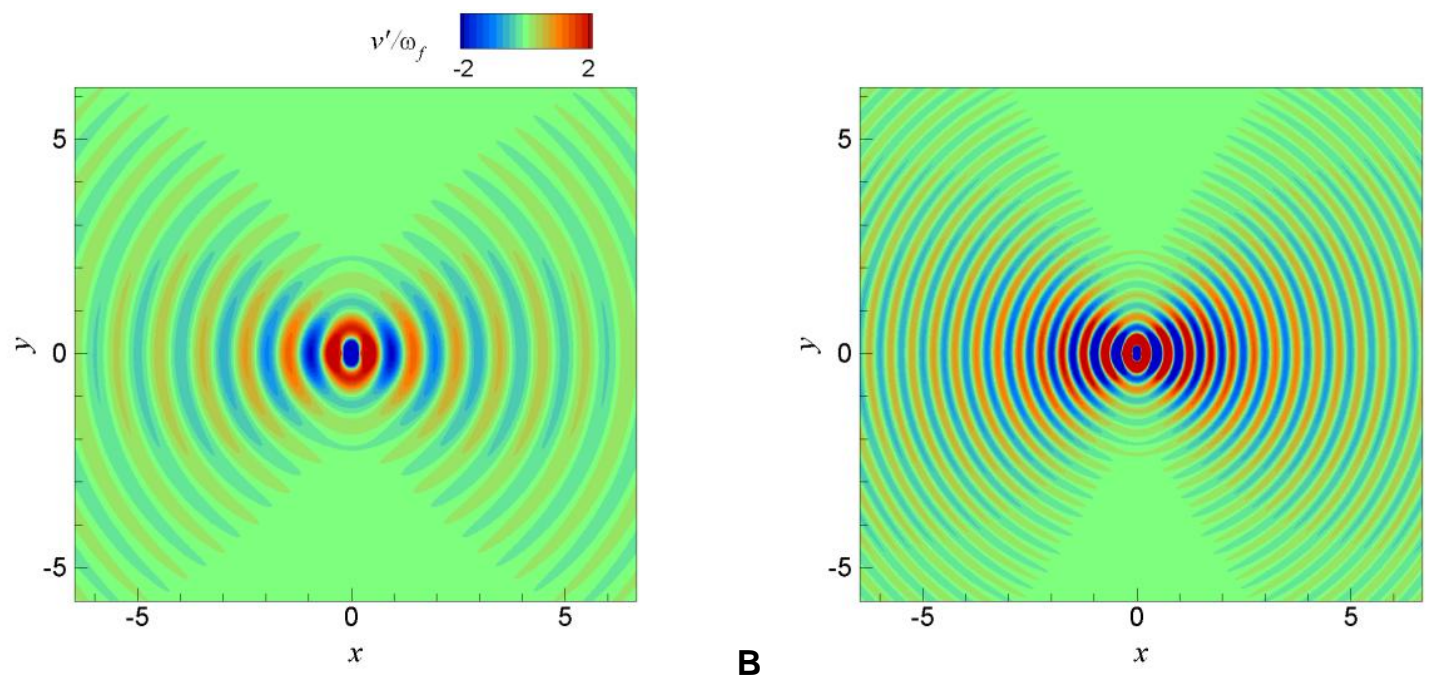

A

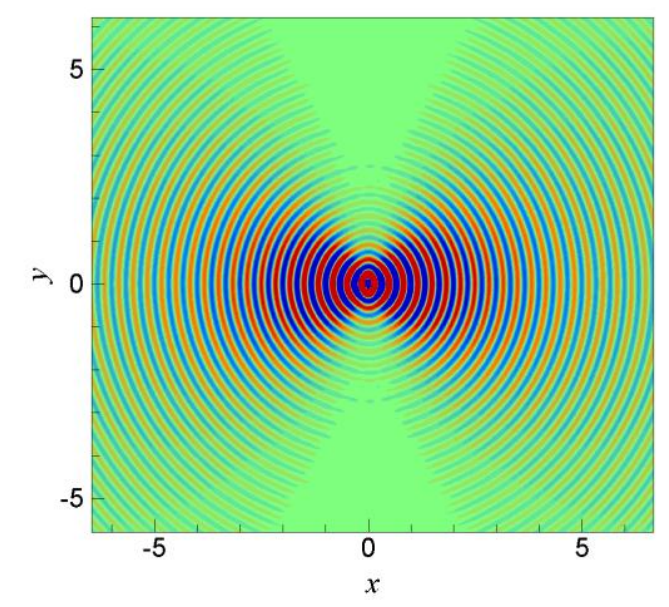

B

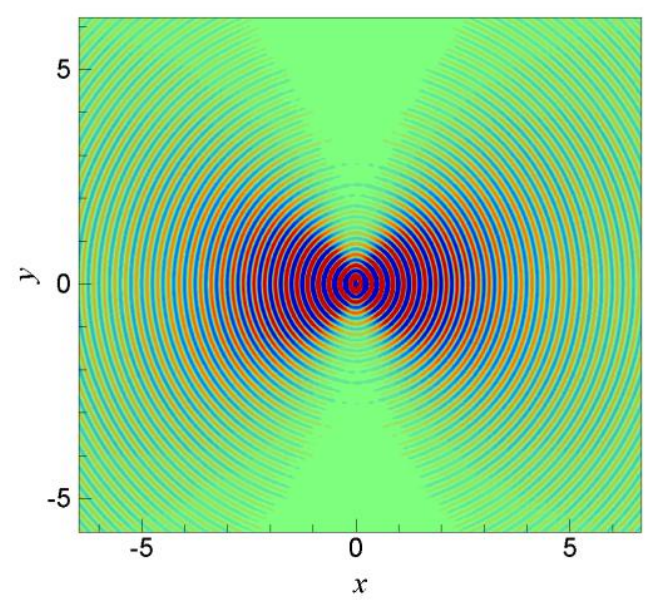

Figure 3: Contours of instantaneous velocity fluctuation in $y$-direction, $v^{\prime}$ divided by the forcing angular frequency, $\omega_{f}$ on the $x-y$ plane $(z=0)$ at $t=100$. A: $\omega_{f}=0.2 \pi$, B: $0.4 \pi$, C: $0.6 \pi$, and D: $0.8 \pi$.

Figure 3 shows the contours of instantaneous velocity fluctuation in $y$-direction, $v^{\prime}$ on the $x-y$ plane. One can see that the directivity of the observed wave is in the $x$-direction, while the forcing is in the $y$-direction. The observed wave lengths are close to the estimated shear wave lengths. The velocity fluctuations, $v^{\prime}$ along the $x$-direction at $y=2$, $z=0$, and $t=100$ are compared with the analytical solutions obtained from Eq. (28) in Fig. 4. The computational results agree well with the analytical solutions, but slight reductions in peak values are observed for $\omega_{f}=0.6 \pi$ and $0.8 \pi$. Note however that since at these frequencies, $\lambda_{s} / \Delta x=8.3$ and 6.25 , respectively, this implies that the shear wave is resolved by about $6-8$ grid points, and the peak loss are therefore caused by the spatial filtering. The results 
of this benchmark problem suggest that the current numerical method can resolve the elastic wave generation and propagation very accurately as long as the wave is resolved with 8 or more grid points per a wave length.
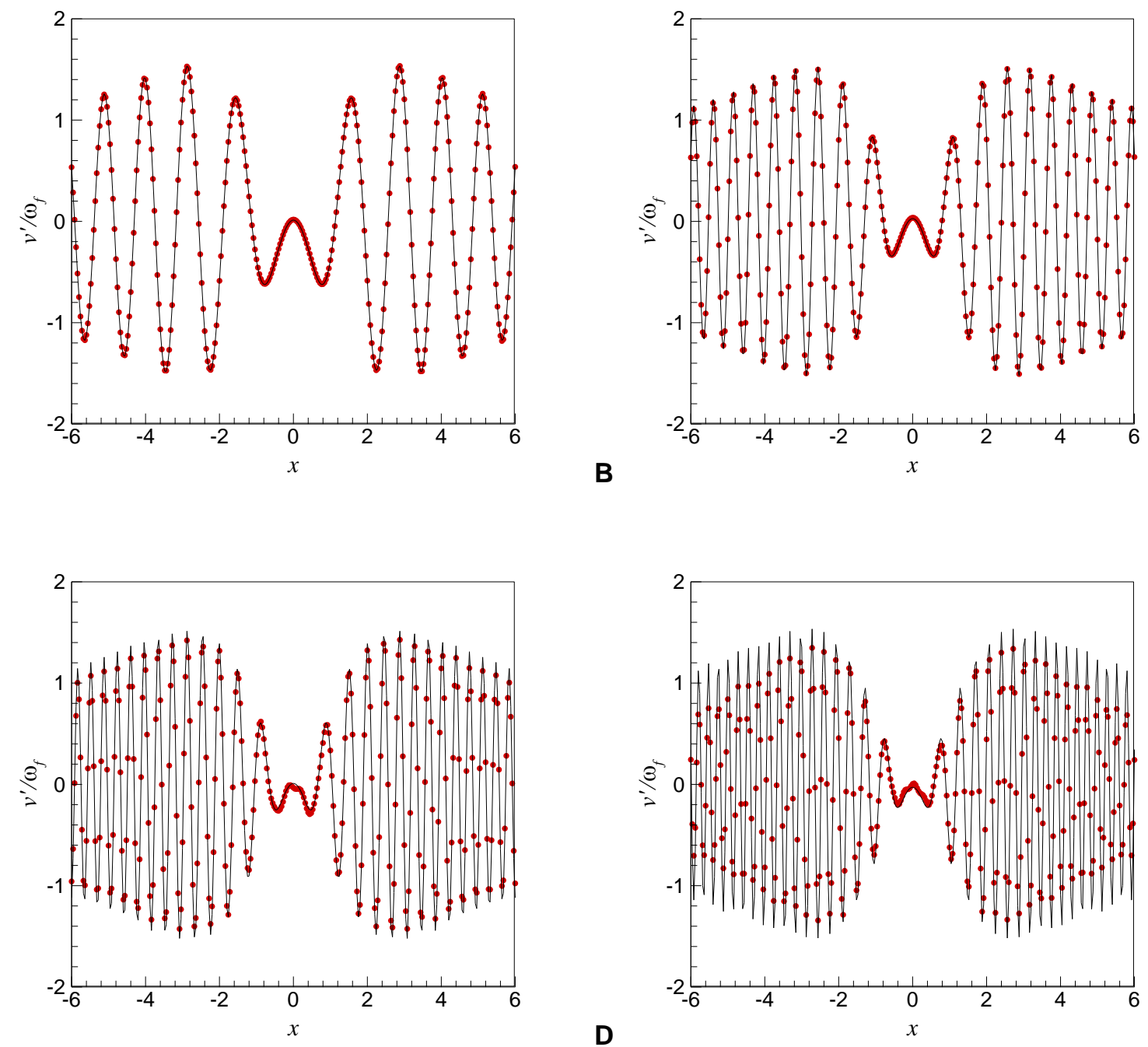

Figure 4: Instantaneous velocity fluctuations, $v^{\prime}$ divided by forcing angular frequency, $\omega_{f}$ along the $x$-direction at $y=2, z=0$, and $t=100$. Symbols: computational result, solid line: analytical solution. A: $\omega_{f}=0.2 \pi, B: 0.4 \pi$, C: $0.6 \pi$, and $D: 0.8 \pi$.

\subsection{Elastic Wave Generated by Surface Pressure Fluctuation on a Sphere}

In order to test the immersed boundary formulation for the traction boundary condition, elastic wave generation by surface pressure fluctuations on a sphere is considered. A schematic of the problem is shown in Fig. 5. The surface pressure on the sphere is fluctuating sinusoidally in time, and this generates an elastic wave that propagates in the radial direction. The analytical solution[46] for the radial velocity fluctuation is given by 


$$
u_{r}^{\prime}(r, t)=\omega_{0} A_{0} k_{p} h_{1}^{(2)}\left(k_{p} r\right) e^{i \omega_{0} t}
$$

where

$$
A_{0}=\frac{P_{0} a^{2}}{(\lambda+2 \mu) k_{p}{ }^{2} a^{2} h_{0}^{(2)}\left(k_{p} a\right)-4 \mu k_{p} a h_{1}^{(2)}\left(k_{p} a\right)},
$$

$a$ is the radius of the sphere, and $h_{n}{ }^{(2)}$ is the spherical Hankel function of the second kind.

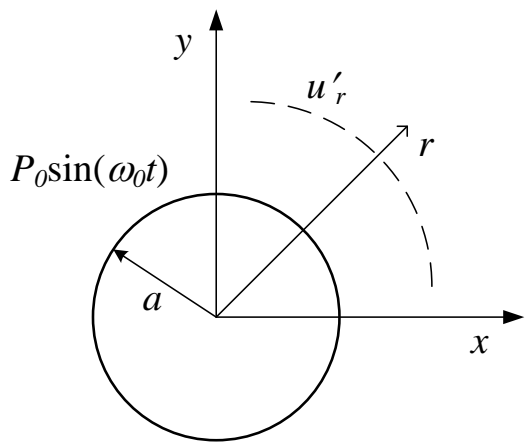

Figure 5: Schematic of the elastic wave generation by the surface pressure fluctuation on the sphere.

The problem is set up with the following parameters; $a=1.0, K=1.0, G=0.1, P_{0}=0.001$, and $\omega_{0}=2 \pi$. For the numerical solution, Eq. (2) is solved on the domain, $-5 \leq(x, y, z) \leq 5$, with the grid spacing, $\Delta x=0.04,0.05,0.08$ and 0.1. The sphere is resolved on the Cartesian grid and the traction boundary condition with the surface pressure is applied by the immersed boundary method (Sec. 2.2.3). For the immersed boundary formulation, $N=2, M=125$, and $l_{\Delta}=\Delta x$ are used. We have found that the second-order polynomial $(N=2)$ is particularly robust for the traction boundary condition.

Instantaneous radial velocity fluctuation computed by the present method with $\Delta x=0.04$ is shown in Fig. 6 . The radial velocity fluctuation is calculated with the Cartesian components by $u_{r}^{\prime}=u^{\prime} x / r+v^{\prime} y / r+w^{\prime} z / r$, where $r=\sqrt{x^{2}+y^{2}+z^{2}}$. The uniform sinusoidal surface pressure fluctuation generates a monopole elastic wave that propagates in radial direction. The computed instantaneous profile of radial velocity fluctuations with various grid resolutions are compared with the analytical solution in Fig. 7A. The computational results agree well with the analytical solution especially for the phase of wave, but slight peak losses are observed for the coarser grid resolutions. Note that with $\Delta x=0.1$, the radius of the sphere is resolved by only 10 grid points. The L2-norm errors of 
numerical solutions are computed against the analytical solution and plotted in Fig. 7B to check the grid convergence. The slope of grid convergence is about 2 , which is expected since the second order polynomial is used for the immersed boundary formulation. In this problem, since the source of wave generation is exactly on the immersed boundary, the overall error is governed by the immersed boundary formulation. The use of high-order compact finite difference scheme, however, improves the dispersion characteristics and helps to capture the correct phase of propagating waves.

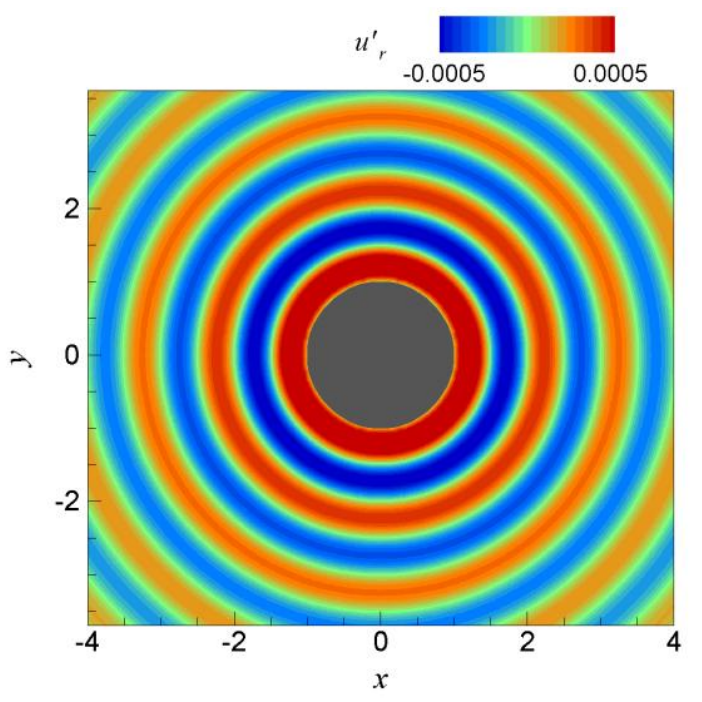

Figure 6: Instantaneous radial velocity fluctuation on the $x-y$ plane at $t=100$.
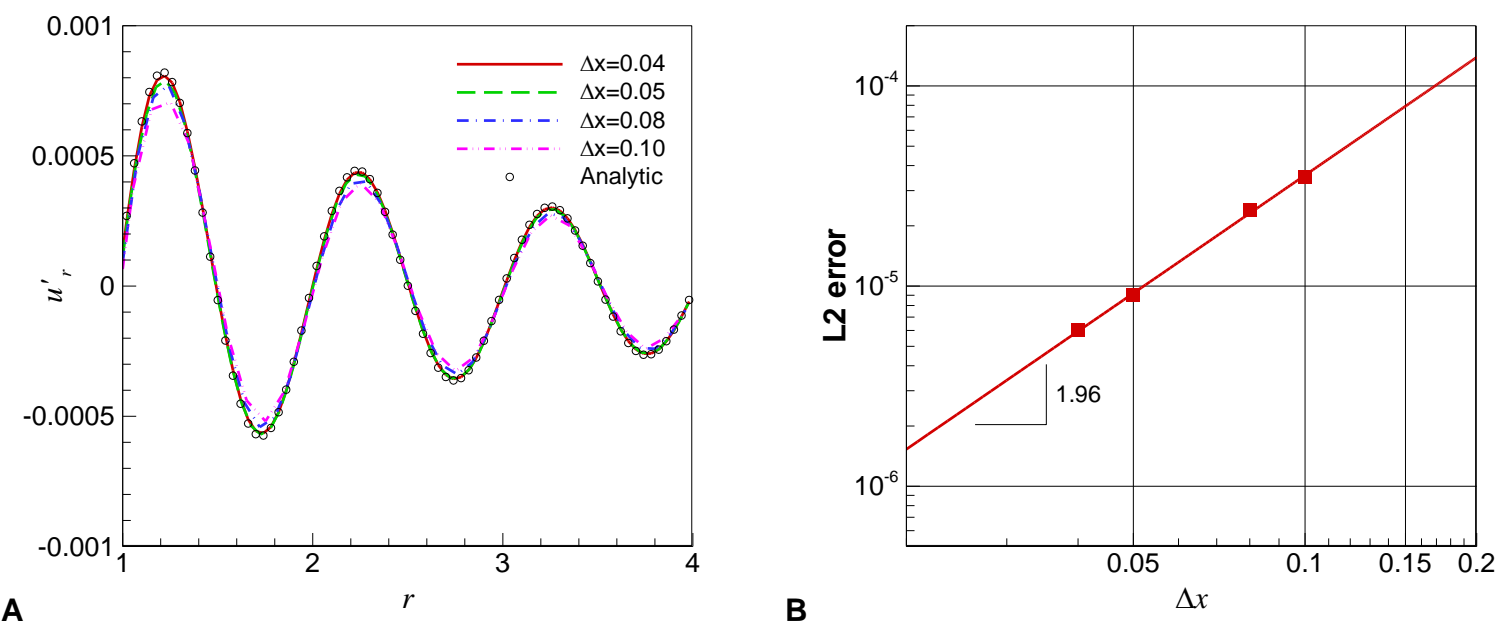

Figure 7: A: Instantaneous radial velocity fluctuation profiles compared with the analytical solution. B: Grid convergence of L2-norm error. 


\subsection{Modeling Aortic Stenosis Murmur}

The murmur generated by an aortic stenosis is well established in the practice of cardiac auscultation [47]. Aortic stenosis is caused by an incomplete opening of the aortic valve and subsequent narrowing of the valve opening area. During systole, the blood flow through this narrowed area results in a strong jet, and due to relatively high flow Reynolds number (about 4000 based on the peak velocity and the aorta diameter), the jet quickly becomes unstable and the shear layer rolls into vortices. The vortices then break into smaller eddies, that generate substantial velocity and pressure fluctuations. The interactions between the vortices and the aortic wall, and the resulting wall pressure fluctuation have long been conjectured to be the source of murmurs. However, the correlation between the blood flow dynamics, wall pressure fluctuations and the intensity and frequency range of the murmur source are not well understood. Furthermore, murmurs are sensed on the surface of the thorax and any fluctuations generated by the blood flow inside the aorta propagate through the thorax to the chest surface wherein they undergo dispersion, reflections and dissipation. In order to understand the characteristics and generation mechanism of such murmurs, it is therefore important to investigate the generation of the elastic wave by the blood flow and its propagation through the biological materials in the thorax.

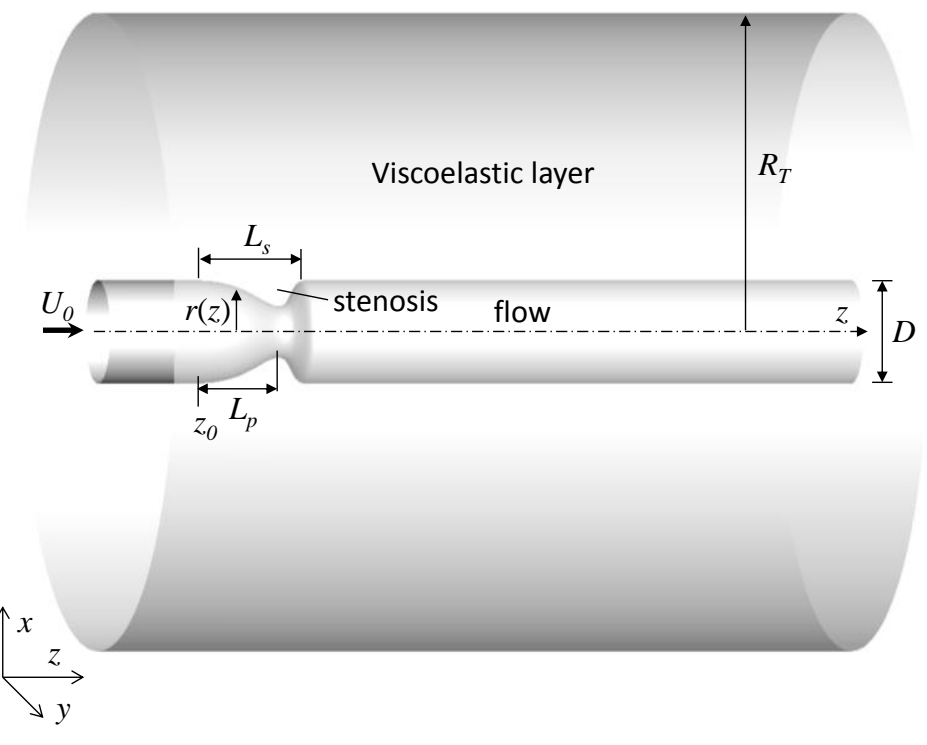

Figure 8: Schematic of aortic stenosis model.

\subsubsection{Simple aortic stenosis model}

In order to investigate the fundamental mechanisms responsible for the generation and propagation of murmurs, we employ a simple model of an aortic stenosis as shown in Fig. 8. This canonical model is designed for computational 
models as well as accompanying laboratory experiments (see Sec. 3.3.4). The model consists of a stenosed (obstructed) flow conduit and a surrounding, cylindrical volume of a homogeneous, viscoelastic material. The flow conduit serves as a model of the aortic stenosis and the surrounding volume of the viscoelastic material is a simple representation of the thorax. The axisymmetric obstruction in the model aorta starts from $1 D$ downstream from the inlet, where $D$ is the diameter of the aorta model, and its shape is given by the following function:

$$
\begin{aligned}
& r(z)=R\left\{1-S \frac{e^{-a\left(z-z_{0}\right)} \sin \left(\pi\left(z-z_{0}\right) / L_{s}\right)}{e^{-a L_{p}} \sin \left(\pi L_{p} / L_{s}\right)}\right\}, \\
& a=\pi / L_{s} / \tan \left(\pi L_{p} / L_{s}\right)
\end{aligned}
$$

where $R=D / 2$ is a unobstructed radius, $S$ is an obstruction ratio, $z_{0}$ is the start position of the stenosis in the axial direction $(z), L_{s}$ is the length of the stenosis, and $L_{p}$ is a distance from the beginning of stenosis to the peak height location. This profile is chosen to model the incomplete opening of the aortic valve with following parameter values: $L_{s}=D, L_{p} / L_{S}=0.8$. The area stenosis ratio (AS) is given by, $1-(1-S)^{2}$, and in the present study, we consider AS=75\% with $S=0.5$, which corresponds to a severe aortic stenosis.

The cylindrical thorax model surrounding the aorta is made up of a homogeneous viscoelastic material and enables the investigation of the generation and propagation of the murmur through a tissue-mimicking medium. In our accompanying laboratory experiment, a silicone gel, ECOFLEX-10 is selected as the tissue mimicking material. The material properties of the ECOFLEX-10 gel are $\rho=1040 \mathrm{~kg} / \mathrm{m}^{3}, K=1.04 \mathrm{GPa}, G=18.39 \mathrm{kPa}$, and $\eta=14 \mathrm{~Pa} \cdot \mathrm{s}$ and these are matched in the computational modeling. The size of the thorax model $\left(R_{T}\right)$ is set to $3.1 D$. Finally, we note that while the flow through the aortic valve is pulsatile, we impose a steady flow through the model. The introduction of flow pulsatility with a well characterized temporal profile is a complexity that the experimental setup cannot currently handle.

\subsubsection{Flow simulation}

The flow simulations are performed only for the region occupied by the flow, i.e. the flow conduit, by solving the incompressible Navier-Stokes equations using the immersed boundary method (Sec. 2.1). The Cartesian computational domain size used is $1 D \times 1 D \times 7 D$ (in $x, y$, and $z$ directions) which covers the flow region inside the stenosed aorta and this is resolved by $128 \times 128 \times 512$ (about $8 \mathrm{M}$ ) grid points. The minimum grid spacing is $1 / 128 D$ and the time step size used is $1.6 \mathrm{e}-3 \mathrm{D} / U_{0}$. Simulations are performed on the TACC-Stampede cluster using 256 CPU cores, and took about $24 \mathrm{hrs}$ wall time for $48 D / U_{0}$. Since aortic murmur is most apparent at peak systole[47], 
the flow simulation is focused on the peak systolic flow phase with a fixed, steady inflow rate. The flow Reynolds number based on the average inflow velocity and the diameter of aorta model is $U_{0} D / v_{0}=4000$, which matches the Reynolds number at the peak systolic flow phase in an adult human aorta.

The inflow to the aorta in-vivo is supposed to be turbulent due to the vortex-dominated nature of the diastolic flow in the left ventricle [48]. In the experiment, the presence of a partially open upstream check-valve also generates a turbulent inflow into the flow conduit. In order to model this inflow turbulence in the simulations, the flow simulations are carried out according to the following procedure: first, the flow in the model aorta is simulated with a uniform, laminar inflow. Once the flow reaches quasi-steady state, the velocity field at the far downstream axial location is averaged in time to obtain a time-averaged velocity profile. The downstream velocity fields are then decomposed into "time-average" and "fluctuating" components by subtracting the time-average velocity profile. The fluctuating components are scaled with a prescribed factor and then added to the time average velocity to generate a scaled "turbulent" inflow velocity profile. This velocity profile is then used for the inflow boundary condition. Note that the fluctuating components are being updated at every time step from the downstream velocity field and thus it provides a time dependent, turbulent inflow condition. This approach is similar to the recycling/rescaling technique used in the turbulent boundary layer simulations[49]. For the present simulation, we extract the downstream velocities at $5 D$ away from the end of stenosis, and the fluctuating components are scaled by $30 \%$ to be used as the inflow fluctuations. After the flow field reaches a quasi-steady state with the turbulent inflow condition, the simulation is conducted for an additional 48 non-dimensional time units for the analysis.

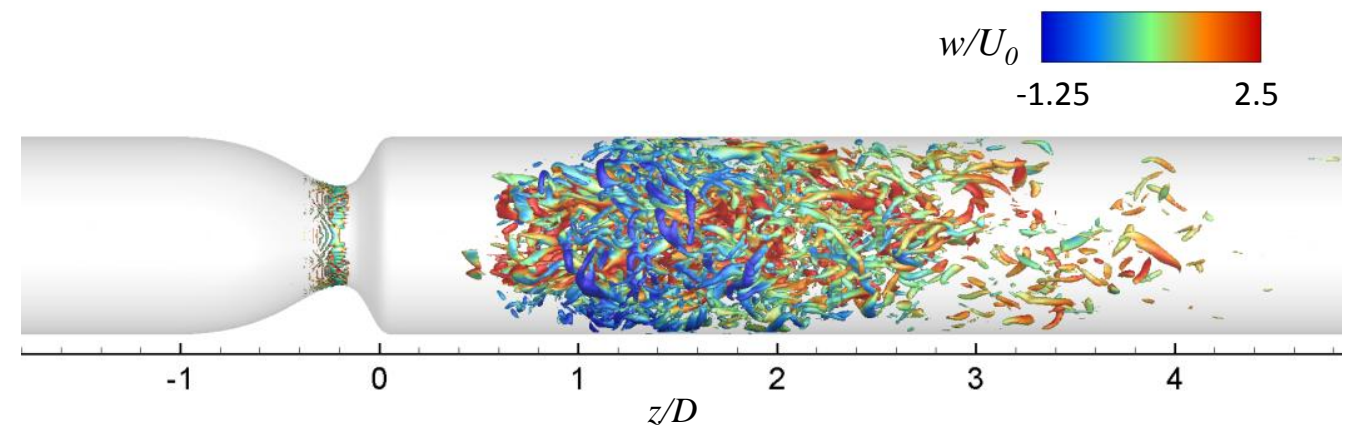

Figure 9: Three dimensional vortex structures visualized by Q-criteria and colored by the axial velocity ( $w)$.

The flow simulation results are visualized in Figs. 9 and 10. Figure 9 shows instantaneous, three dimensional vortex structures represented by the Q-criteria [50] and a conglomeration of vortex structures are clearly observed to 
be centered around $z / D=1.5$. These vortices are also found to dissipate rapidly beyond $z / D=3.0$ and are mostly gone beyond $z / D=4$.0. Instantaneous flow fields on a central $x-z$ plane are shown in Fig. 10. The flow through the stenosis generates a strong jet with a distinct shear layer around the jet. The shear layer becomes unstable and rolls into vortices at around $z / D=1$. The vortices then interact with the wall as well as the other vortices in the shear later and break into smaller eddies. These vortices are also regions of low pressure (Fig. 10) and the dynamic interaction of these vortices with one another and with the wall is responsible for the generation of wall pressure fluctuations. Much of this wall interaction occurs near the reattachment point, which is around $z / D=1.5$.

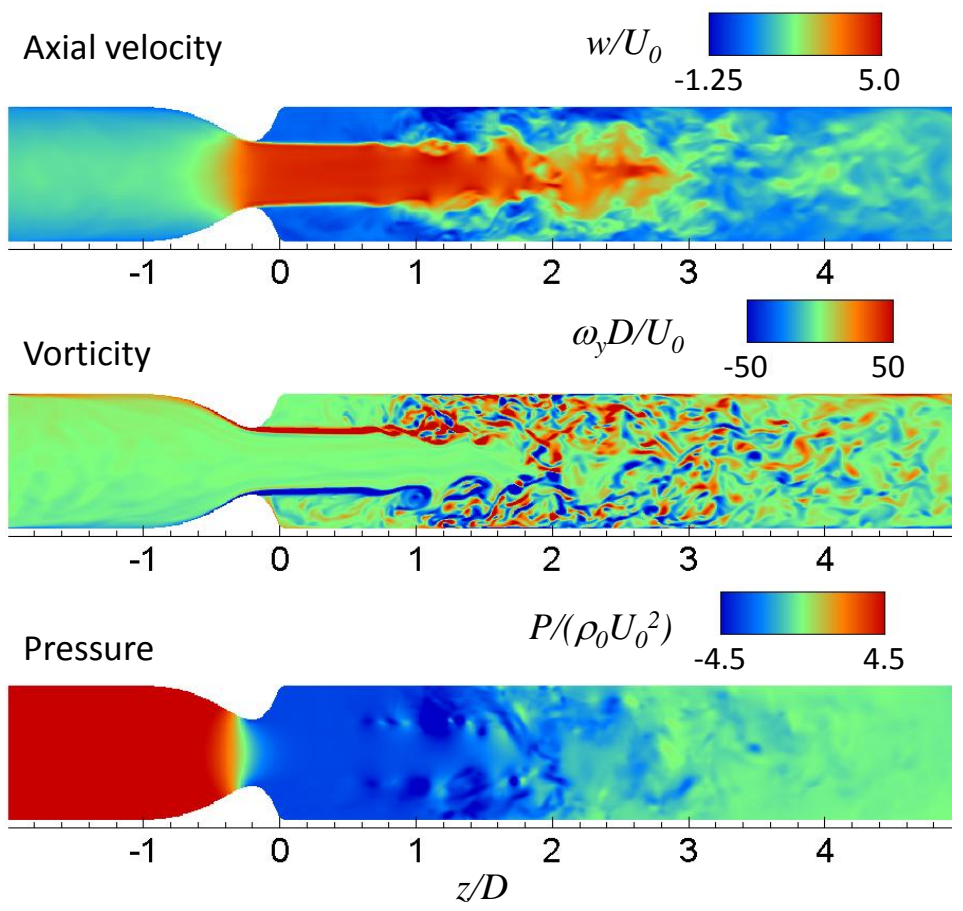

Figure 10: Instantaneous flow fields on the cross section at the $x-z$ plane.

In the previous studies $[12,14-16]$, it has been suggested that the wall pressure fluctuations are the main source of murmur generation. The wall pressure fluctuations are, therefore, monitored at various axial locations to identify the source region. The frequency spectra of wall pressure fluctuations are plotted in Fig. 11. Strong pressure fluctuations are observed at downstream locations between $z / D=1$ and 3, where the shear layer rolls into vortices that are interact with the wall, while the fluctuations are weak at the upstream locations (between $-1 D$ and 0 ). Comparing the spectra at the upstream and downstream locations, one can see that a significant increase of spectral energy 
occurs for the Strouhal number $(\mathrm{St}=f D / U$ ) range between 1 and 10. As the vortices dissipate further downstream $(z / D>4)$, the amplitude of pressure fluctuation decreases significantly. The strongest wall pressure fluctuation is observed at around $2 D$ downstream of the stenosis. The spectrum is broad-band in its shape and shows an almost flat plateau for Strouhal number lower than 1.5; beyond that, it slowly decays for the higher frequencies. For the frequency range $1<\mathrm{St}<10$, the strength of pressure fluctuation at $2 D$ downstream is about two orders-of-magnitude higher than ones at the upstream locations. Based on these observations, the major source of murmur is expected to be at around $2 D$ downstream from the end of stenosis.

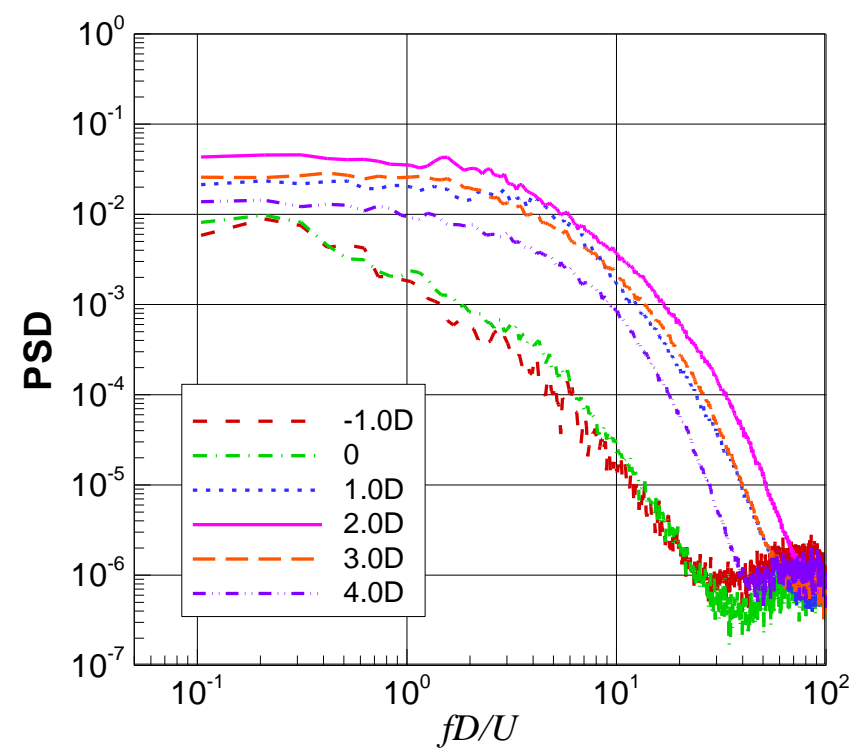

Figure 11: Power spectral densities of wall pressure fluctuations at the axial locations from $-1 D$ upstream to $4 D$ downstream from the end of stenosis $(z=0)$.

\subsubsection{Simulation of the Murmur}

The generation and propagation of elastic waves by the flow fluctuations are simulated by solving the linear elastic wave equations, Eq. (2) in the surrounding viscoelastic layer shown in Fig. 8. The geometries representing the lumen boundary and the tissue layer are immersed into the Cartesian domain of size $13 D \times 13 D \times 14 D$, which matches the experiments. The computational domain is resolved by $200 \times 200 \times 320(12.8 \mathrm{M})$ grid points and the minimum grid spacing is $0.04375 D$. For the IBM formulation, the parameters employed are $N=2, M=125$, and $l_{\Delta}=0.05 D$. The domain is decomposed into $8 \times 8 \times 16$ (1024) sub-domains for parallel computation and a total of 1024 CPU cores are 
used in the TACC-Stampede cluster. The simulation for 36 non-dimensional time units takes about 60 wall-time hours (61440 CPU hrs).

Since the characteristic speed of the elastic compression wave in soft-tissue is much faster $\left(c_{b}=1000 \mathrm{~m} / \mathrm{s}\right.$, $U_{0} / c_{b}=2.5 \times 10^{-4}$ for the present case) than the flow speed, this severely restricts the time-step size for solving the linear structural wave equations. To alleviate this numerical difficulty, in the present study, the compression wave speed is arbitrary decreased by an order of magnitude to be $U_{0} / c_{b}=2.5 \times 10^{-3}$, while the shear wave speed is kept the same $\left(U_{0} / c_{s}=5.9 \times 10^{-2}\right)$. Note that even with this decreased compression wave speed, the wave length corresponding to frequencies with $\mathrm{St}<10$ are longer than $40 \mathrm{D}$, which significantly exceeds the longest geometrical length scale of the present problem $(7 D)$. Thus, the effect of this change on the scattering of the compression elastic wave should be quite small. A similar approach has been employed successfully in the previous studies[16, 51]. We have also confirmed using the canonical case of the elastic wave propagation due to a point force (Eq. 26) that the one orderof-magnitude decrease in $c_{b}$ has little effect on the elastic wave propagation result for the frequency range $\mathrm{St}<100$, which is the relevant range for these murmurs.

The wall pressure fluctuations obtained from the flow simulation are recorded and used for the time dependent boundary conditions (Eqs. 5 and 6) at the interface between the flow and tissue region. The time step size used for the wave simulation is $0.016 \mathrm{D} / \mathrm{c}_{b}\left(4 \times 10^{-5} \mathrm{D} / \mathrm{U}_{0}\right)$, which is 40 times smaller than the time step size of the flow simulation. The recorded surface pressure fluctuation data is therefore interpolated in time using a second order Lagrange interpolation. At the outer radial boundaries of the tissue layer, a traction free condition (Eq. 4) is applied and a zero displacement, wall boundary condition is applied at the two ends.

The simulation results for the propagation of the elastic wave in the tissue are presented in Figs. 12 and 13. The pressure fluctuation at the lumen boundary (interface between the blood flow and tissue layer domains) generates elastic stress fluctuations, which are coupled with velocity fluctuations in the tissue mimicking gel. The resulting elastic waves propagates outward and are reflected at both the wall and the traction free boundaries. Since there is no traction on the outer boundary of the tissue phantom, the stresses are necessarily zero there, but the outer surface vibrates with the velocity fluctuations of the elastic waves. Since the stethoscope mainly senses the wall normal component of the surface vibration and converts it into audible sounds[14] it is the surface normal velocity fluctuation that is primarily responsible for generating the audible murmur. In the present case, since the tissue phantom is cylindrical, the surface normal component is a radial velocity fluctuation that can be calculated as 
$u_{r}^{\prime}=u^{\prime} x / r+v^{\prime} y / r$, where $r=\sqrt{x^{2}+y^{2}}$. In figure 12 , the instantaneous radial velocity fluctuation contours are plotted on several cross sectional planes. One can clearly see the wave generation from the lumen boundary and its propagation to the outer boundaries.

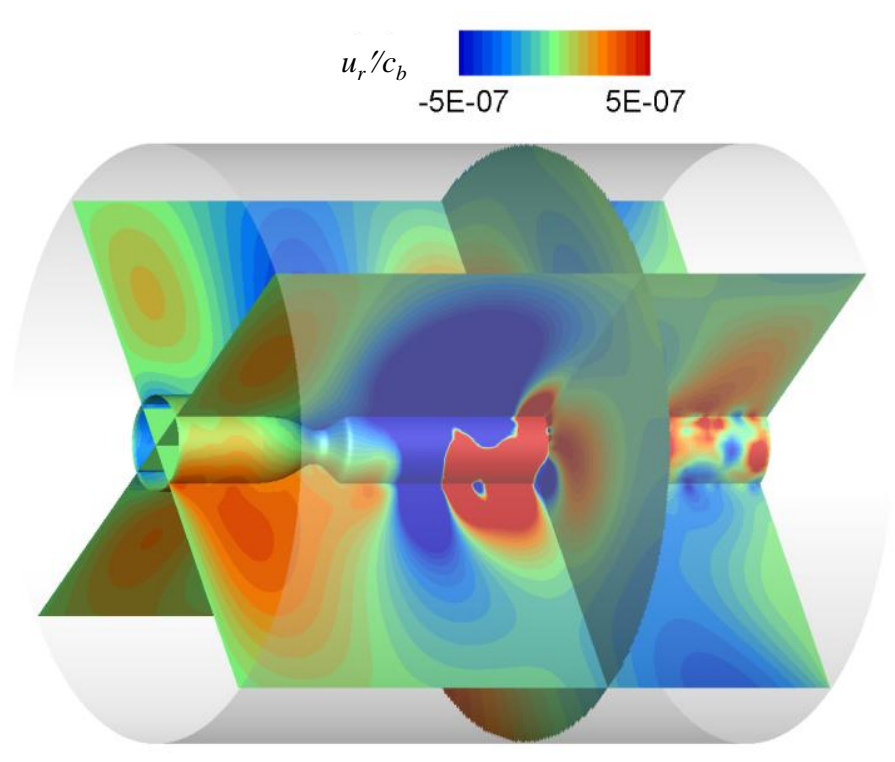

Figure 12: Instantaneous radial velocity fluctuation contours at cross section planes.

Stress and velocity fluctuation components are examined on the $x-z$ cross plane at the center of tissue phantom. Figures $13 \mathrm{~A}$ and 13B show the normal stress fluctuation in $x$-direction and shear stress fluctuation, respectively. Note that the $x$-direction in these plots is normal to the outer boundary surface. It is observed that the strong normal stress originates at the lumen boundary at an axial location between $z / D=1$ and 2 , where the hemodynamic wall pressure fluctuation is the strongest. A noticeably high shear-stress fluctuation $\left(p_{x z}^{\prime}\right)$ is also observed at the same location, and the figures show that the waves of these stresses propagate to the outer boundary where they vanish because of the traction free condition at the outer boundary. The propagation of elastic waves is more clearly visible for the velocity fluctuation contours in Figs. 13C and 13D. Figure 13C shows the fluctuation of velocity component normal to the outer boundary, while the tangential component is shown in 13D. One can see that the origin of propagating wave structure is located at the lumen boundary with the axial location between $z / D=1$ and 2 . We also note that the outer boundary surface is fluctuating not only in the normal direction but also in the tangential direction. 

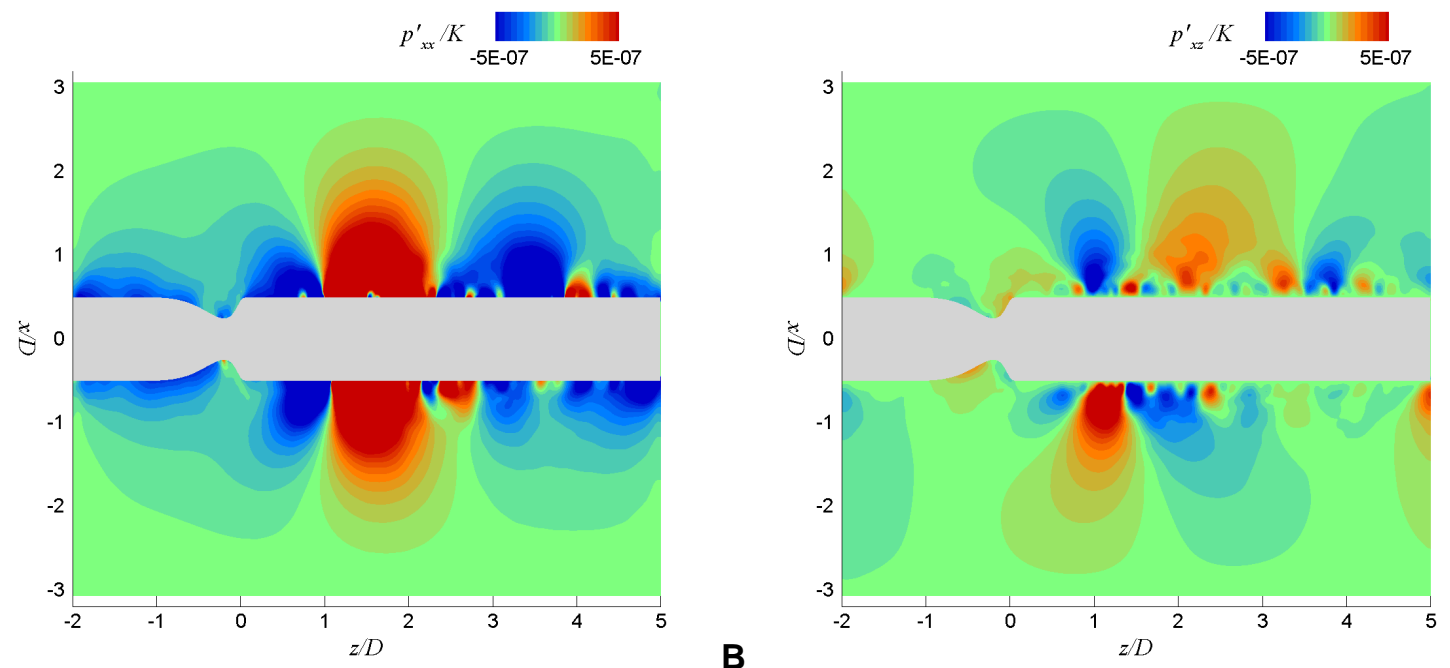

A

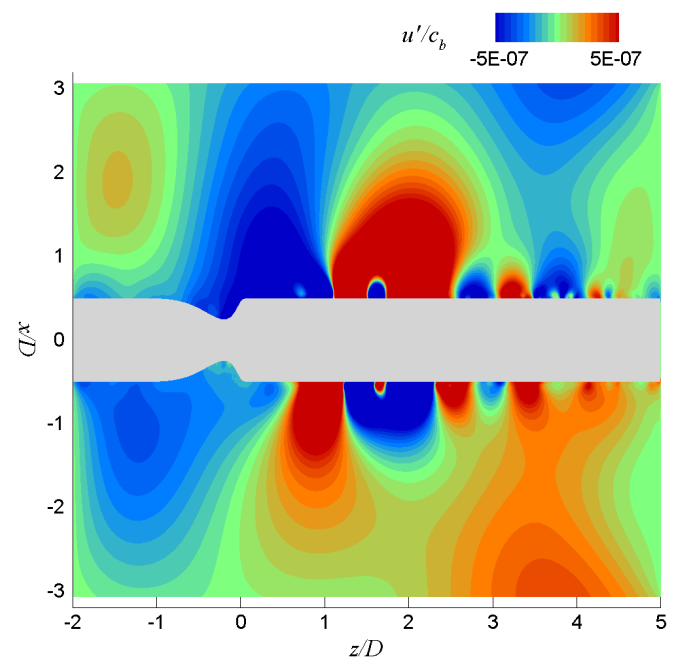

B

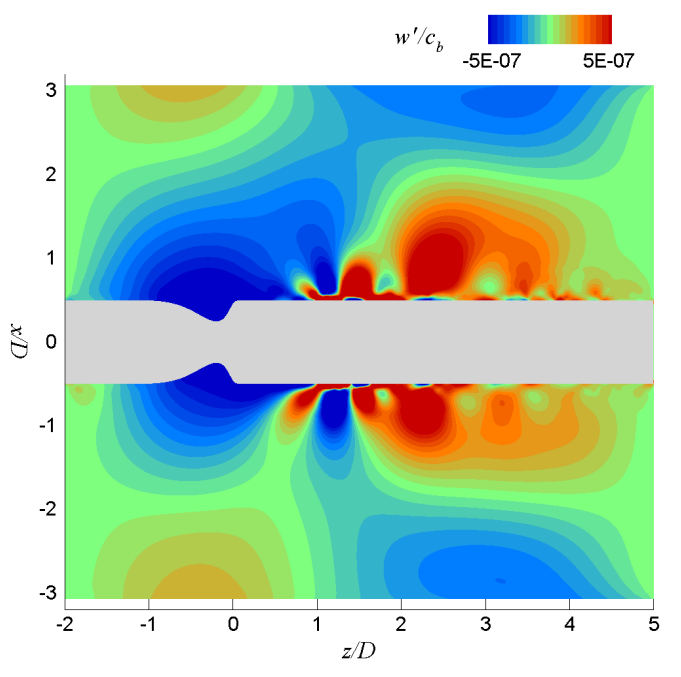

Figure 13: Instantaneous elastic wave fields in $x-z$ center plane. A: fluctuating normal stress in $x$-dir (vertical). B: fluctuating shear stress. C: $x$-dir (vertical) velocity fluctuation. D: $z$-dir (horizontal) velocity fluctuation.

To analyze the dominant wavelength of the acoustic wave in the axial direction, instantaneous profiles of the stress and velocity fluctuations are extracted along the $z$-direction at $x=2 D$, and plotted in Fig. 14A, and these exhibit clear wave-like structures. The wave number analysis done by the Fourier transform is presented in Fig. 14B and the result shows that the dominant wave number is about $k_{z} D=1.82$ except for the axial velocity fluctuation. This dominant wave number corresponds to a wavelength of about 3.45D. Since the estimated wavelength of the compression wave for $\mathrm{St}<10$ is longer than $40 D$, this dominant wave structure is expected to be caused by the shear wave. For the shear wave, the wavelength of $3.45 \mathrm{D}$ corresponds to a non-dimensional frequency, $\mathrm{St}=4.9$. 

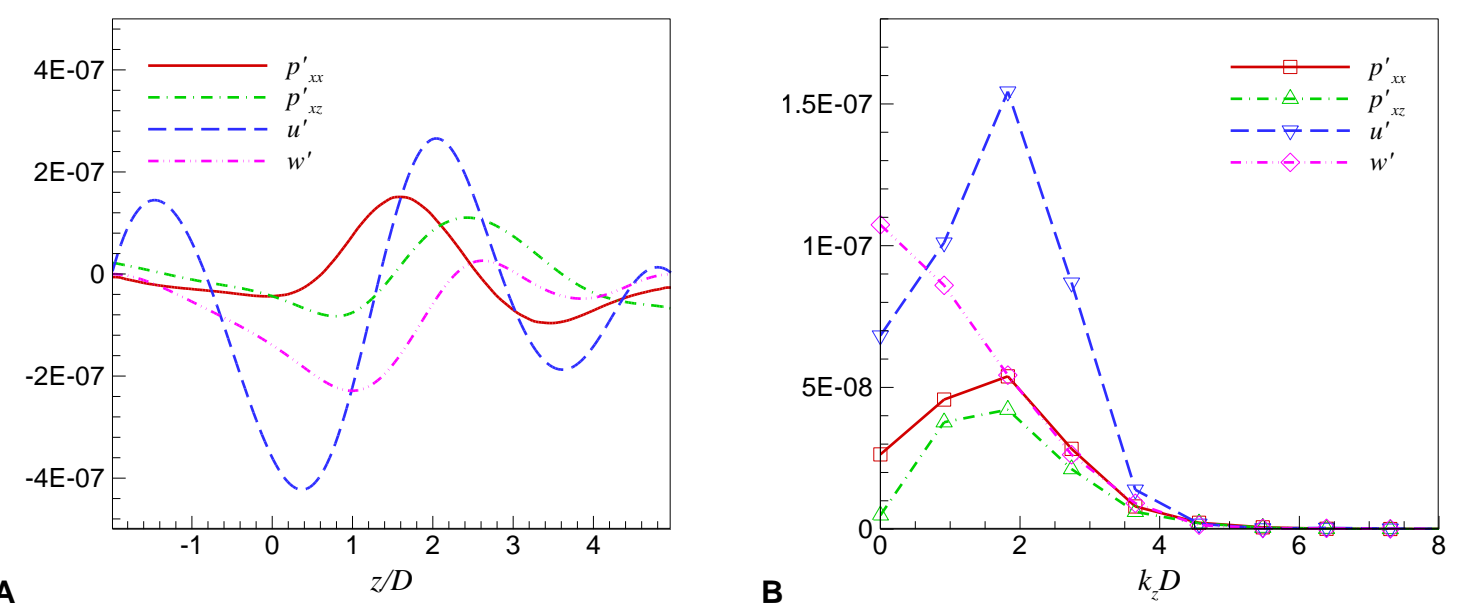

Figure 14: A: Instantaneous profiles of stress and velocity fluctuations at $x=2 D$. B: Wave number analysis using the Fourier transform.

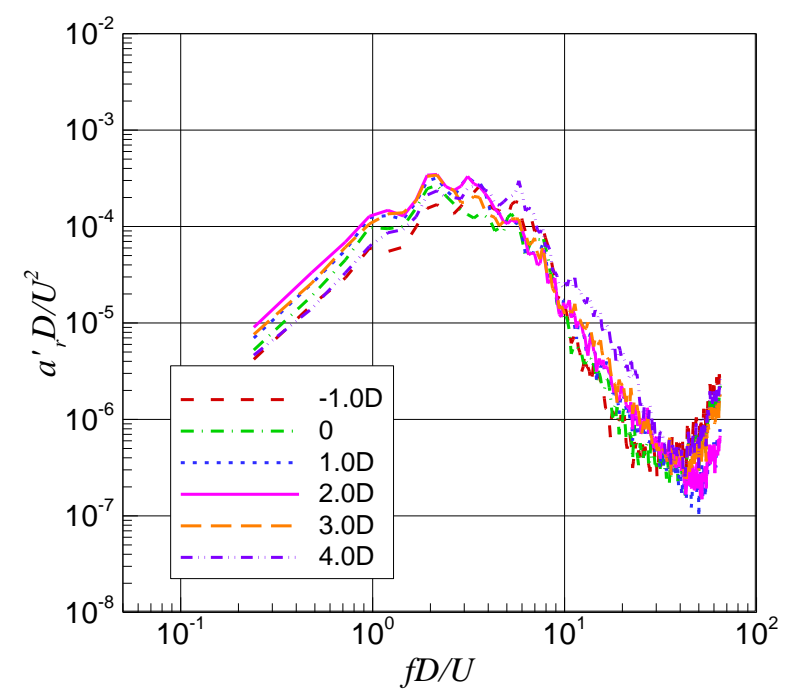

Figure 15: Frequency spectra of radial accelerations monitored on the outer surface of tissue layer at the axial locations from $-1 D$ upstream to $4 D$ downstream from the end of stenosis $(z=0)$.

Based on the type of stethoscope or sensor employed, the murmur measured on the outer surface can be related to the surface normal velocity or the acceleration[14]. In our companion experiment, we measured the surface fluctuation using accelerometers, and thus, in the simulation, we monitor the surface normal (radial) acceleration $\left(a_{r}{ }_{r}=\partial u^{\prime}{ }_{r} / \partial t\right)$ at the various axial locations on the outer surface of the tissue layer. The frequency spectra of the 
radial acceleration are presented in Fig. 15. The spectra are ensemble averaged along the circumferential direction, since the problem can be considered statistically axi-symmetric.

The shape of spectrum for the radial acceleration does not change much for the different axial locations because the wave generated from the lumen wall $(z / D=1-2)$ has a direct line-of-sight to all the axial locations as shown in Fig. 9. The spectral shape can be characterized as follows: for frequencies for which $\mathrm{St}<1$, the spectral amplitude decreases for the lower frequency. The spectrum shows a plateau with a few peaks in the range $1.5<\mathrm{St}<5$, and the amplitude decays rapidly for the higher frequencies, $\mathrm{St}>6$. Note that the frequency, $\mathrm{St}=4.9$ associated with the dominant shear wavelength, $3.45 \mathrm{D}$ (Fig. 14) falls within the frequency plateau range $(1.5<\mathrm{St}<5)$, and this supports the notion that the dominant wave structure observed in Fig. 13 is generated by the shear wave mechanism.

While the shape does not change much, the amplitude of spectrum varies noticeably with axial location. The amplitude is the highest for the axial location of $z=1 D-2 D$, and it decreases for the upstream and downstream locations. This observation agrees with the fact that the hemodynamic wall pressure fluctuation is strongest at $z=1$ $2 D$, and also, as one can see in Fig. 13, the dominant wave structure originates at the same axial location. This suggests that the elastic wave responsible for the murmur (which we contend is the shear wave for the present case) is mainly generated from the location of the strongest hemodynamic pressure fluctuation, which agrees with the finding from the previous study[16]. It is interesting to note however that the spectrum at the further downstream location $(z=4 D)$ exhibits more energy for the higher frequencies $(\mathrm{St}>6)$ and this may be due to the interaction between the high wave number structures and the zero-displacement wall boundary condition, as seen in Fig. 13.

\subsubsection{Comparison with experimental measurements}

A laboratory experiment has been performed for the same set-up with the computational model. The experimental set-up is shown in Fig.16. As mentioned above, the viscoelastic tissue phantom is made with the silicone gel ECOFLEX-10 and the flow conduit with the stenosis is created using a rapid prototyping, 3D printed mold inside the gel sample. The diameter of the gel sample is $D=1.5875 \mathrm{~cm}(5 / 8 \mathrm{inch})$. The top and bottom of the gel phantom are fixed to acrylic plates, while the radial boundary remains free. The flow conduit is connected to a flow circuit which provides a constant flow of water, with an average flow speed of $U=0.25 \mathrm{~m} / \mathrm{sec}$. The resulting flow Reynolds number is about 4000 . The experiments are performed in an anechoic chamber and the normal acceleration of outer surface vibration is measured by an accelerometer (HP) and a contact microphone (BIOPAC) (see Fig. 16). Both 
sensors are designed for the use in biomedical research. More details about the experiment can be found in the Ref.[18].
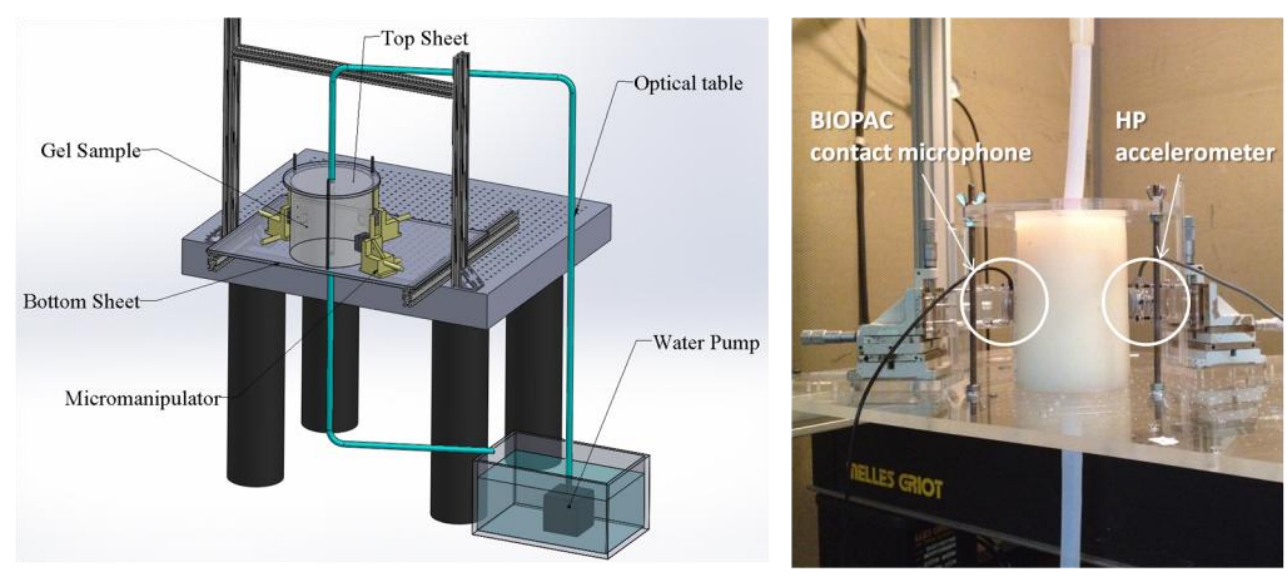

Figure 16: Experimental set-up for the murmur generation and measurement.

The frequency spectra of radial accelerations at the axial location of $z=2 D$ measured from the experiments are compared with the simulation result in Fig. 17A. Note that the data from the simulation is converted to dimensional values for the comparison with the experimental data. Since the HP and BIOPAC sensors have different gains, the amplitudes are adjusted match each other. Also, it is noted that neither sensor is able to accurately detect frequencies below $15 \mathrm{~Hz}$. Figure 17A shows a reasonably good agreement between the simulation and experimental results. A plateau in the spectrum in the frequency range between 20 and $80 \mathrm{~Hz}$ (corresponding to $1.5<\mathrm{St}<5$, approximately) appears in both the simulation and the measurements, and the rate of decay of the amplitude for the higher frequencies also observed to match well. Overall the spectral shape predicted by the simulation coincides very well with the measured data. Although not shown here, the frequency spectra at other axial locations shows a similar degree of agreement.

Validation of the computational model has been extended beyond the comparison of spectra at selected locations. Since one of the long-term objectives of the present study is to use these computational models to create detailed acoustic "maps" of heart murmurs and to eventually use this information for automated source localization, it is essential to show that the computational modeling procedure is capable of accurately reproducing the spatial variation of the acoustic energy on the surface. In order to assess this, the axial variations of the signal amplitudes on the surface are also compared. The spectral energy of the signal in a given frequency range is calculated as follows: 


$$
E_{f 1-f 2}(z)=\int_{f 1}^{f 2}\left|a_{r}^{\prime}(f, z)\right|^{2} d f
$$

The axial variations of the above spectral energy are examined for three frequency bands: 30-60 Hz (low), 60-120 $\mathrm{Hz}$ (mid), and 120-480 $\mathrm{Hz}$ (high frequency) as well as the total range from $30-480 \mathrm{~Hz}$. These spectral energy mappings are presented in Fig. 17B for the simulation and experiment. In the experiment, the mapping is performed with the HP sensor only, since the probe of BIOPAC sensor is too large to resolve the axial variation of the amplitudes. To account for the errors and uncertainties in the experiments, the measurements are repeated multiple times and the mean values and error bars are presented in Fig. 17B.
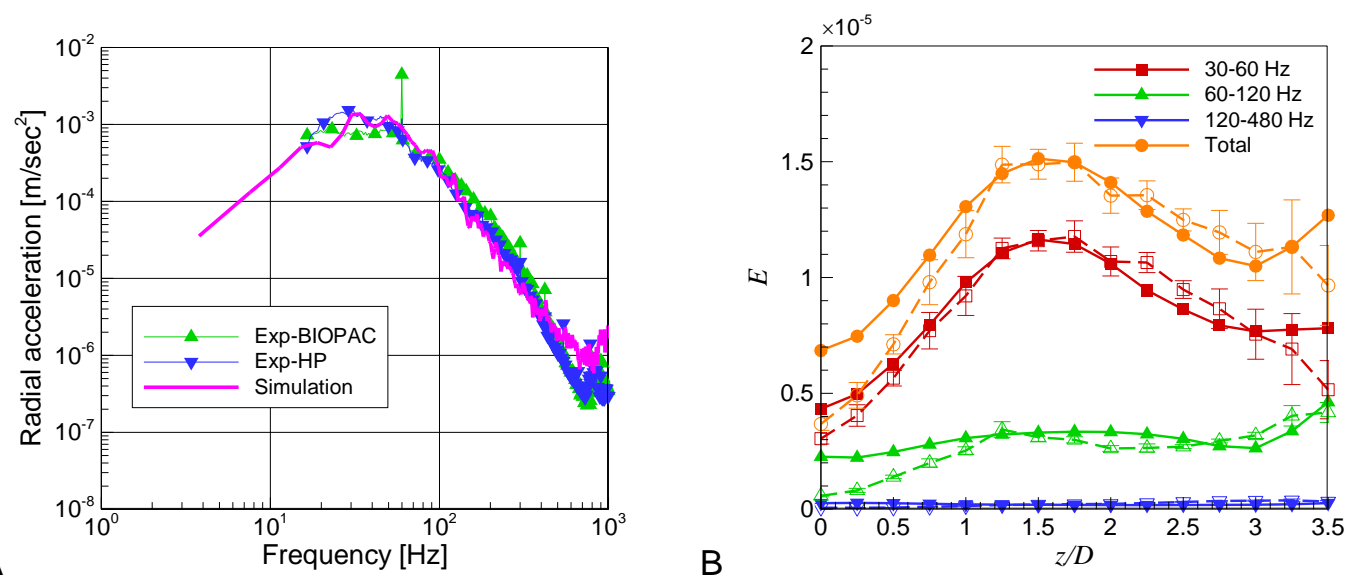

Figure 17: Comparisons between the simulation results and the experimental measurements. A: Frequency spectra of radial accelerations measured on the outer surface of tissue layer at the axial location of $2 \mathrm{D}$ downstream from the end of stenosis $(z=0)$, Exp-BIOPAC: measurement by BIOPAC contact microphone, Exp-HP: measurement by HP accelerometer. B: Variation of spectral energy of the signal for three different frequency bands; solid line with filled symbol: simulation; dashed line with hollow symbol and error bar: experimental measurement.

The figure shows a reasonably good agreement between the simulation and experimental results. The peak energy for $30-60 \mathrm{~Hz}$ band is found at the axial location between $z=1 D$ and $2 D$ for both the simulation and experimental results, and this confirms the notion that the primary source of murmur is located on the lumen at that axial location. The axial variation profiles of the energy for $30-60 \mathrm{~Hz}$ low-frequency band from the simulation and measurement also show a reasonably good match except in the region beyond $z=3 D$, where the simulation show a higher energy content than the experiments. With regard to this discrepancy between the simulation and experiment 
beyond $z=3 D$, we note that the scatter in the experimental data is generally larger for these locations indicating additional effects such as complex wave transmission into the bottom plate in the experiments, which are not fully accounted for in the simulations. Furthermore, we note that there is some gravitational settling in the silicone gel which affects the radius as well as density of the phantom for the downstream portions of the gel; this effect is not modeled in the simulation. The profiles of the energy for mid frequency band (60-120 Hz) show acceptable agreement between the simulation and measurement, but slight discrepancy is observed for $z<1 D$. For the high frequency band $(120-480 \mathrm{~Hz})$, the overall energy content is quite low but well matched between the simulation and experiment. Thus, the predictions for the simulations can be considered reasonable over the entire frequency range of interest.

The reasonably good agreement between the simulation and the experimental measurement observed here indicates that the key assumptions underlying the murmur modeling procedure, such as (a) the use of the hemodynamic wall pressure fluctuations as the primary sources of the elastic wave generation; (b) the use of the linear viscoelastic wave equation (Eq. 2) to model the elastic wave propagation and (c) the numerically expedient approach of reducing the compression wave speed, are all reasonable. It also indicates that the computational method employed to solve the governing equations is well suited for modeling the physics of heart murmurs.

\subsubsection{Analysis of Murmur Signals}

The reasonable agreement between the simulations and experiments provides confidence in the current modeling approach and the simulations results can now be examined in detail to obtain insights into the physics of murmur propagation. In the arena of hydroacoustics (or low speed aeroacoustics)[52], an alternative, and efficient way to estimate the flow-induced sound is the combination of flow simulation results and the analytical solution of the wave propagation[53]. Since such analytical solutions are written in terms of various source mechanisms, they can provide further insights into the physics of the problem. In this section, we therefore estimate the murmur by combining the flow simulation result and an analytical tool. One analytical tool that is well suited for use in conjunction with the computational model is the free space Green's tensor (Eq. 26). One advantage of this approach is that the terms associated with compression and shear components of the elastic wave associated with the murmur can be evaluated separately (the first and second terms on the right-hand-side of Eq. 26 represent the compression and shear wave components of the elastic wave corresponding to the murmur propagation), and thus, it is possible to 
estimate the relative importance of each mechanism. To use the free space Green's tensor, the current problem is simplified as shown in Fig. 18. The wall pressure fluctuation at the lumen boundary is replaced with a series of discrete point forces, and the final solution at the observation point is obtained by the superposition of contributions from all these point forces:

$$
\hat{a}_{2}(\omega)=2 \sum_{k}(-i \omega)^{2} G_{2 j}\left(\vec{r}_{k}, \omega\right) F_{j, k}(\omega), \quad F_{j, k}(\omega)=n_{j, k} \hat{P}_{k}(\omega) \Delta A
$$

where $\hat{a}_{2}(\omega)$ is the radial acceleration in the frequency domain, $k$ is the index for the discrete point forces, $F_{j, k}$ is the normal force at the lumen boundary due to the hemodynamic pressure $\hat{P}_{k}(\omega)$, and $n_{j, k}$ is the unit vector normal to the lumen wall. We used $30 \times 16$ ( 30 points in the circumferential direction and 16 points in the axial direction) discrete point forces equally distributed on the lumen wall between $z=0$ to $4 D$ and thus $\Delta A=\pi D^{2} / 60$. Note that Eq. (33) does not include effects associated with the generation of the surface wave. A previous study[20], has shown that for a tissue like materials and especially at low frequencies, the surface wave contribution is weak and the free space solution agrees reasonably well with the full analytical solution. The multiplication by a factor of two in Eq. (33) is to take into account the reflection at the traction free boundary as suggested in Ref.[20]. The frequency spectrum of the radial acceleration at $z / D=2$ evaluated by using Eq. (33) is compared with the full 3D simulation result. The analytical accelerations are also estimated separately for the compression and shear waves, and the corresponding spectra are presented in Fig. 19.

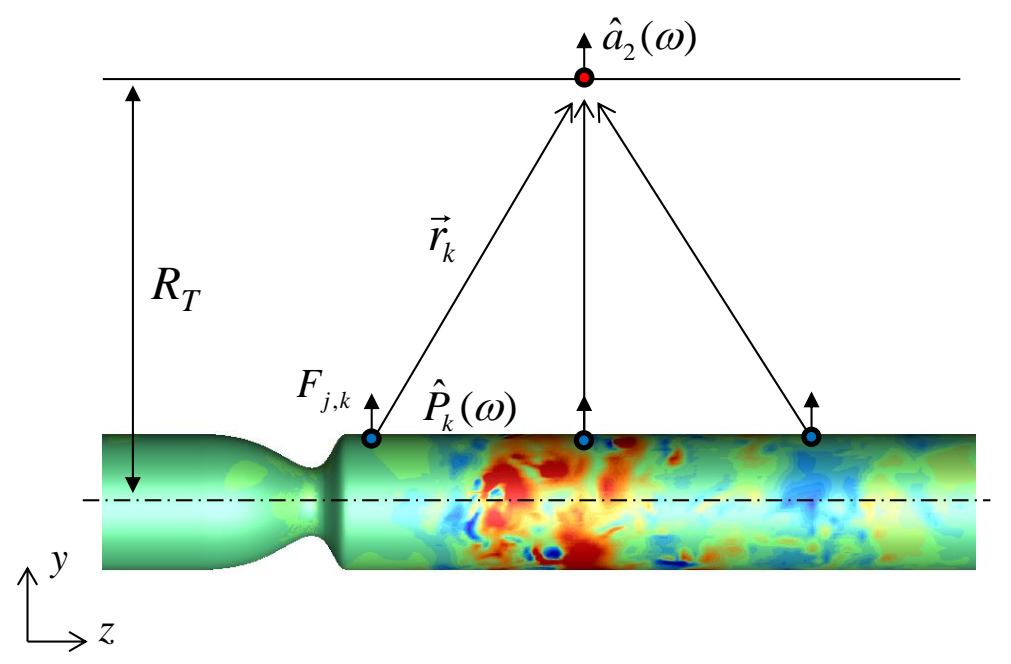

Figure 18: Schematic of analytical evaluation of the murmur. 
A number of interesting observations can be made regarding the comparison in Fig. 19. We note that the frequency spectrum of the analytical evaluation (Green-Total) matches reasonably well with the full 3D simulation result. The key characteristics of the spectral shape - the decrease in amplitude for $\mathrm{St}<1$, a plateau at $1.5<\mathrm{St}<5$, and decay for higher frequencies are all represented well in the Green's tensor solution. Since the Green's tensor model solution agrees with the full simulation result, the effects of compression and shear waves can be analyzed based on the Green's tensor solution. We find that at very low frequencies $(\mathrm{St}<1)$, the amplitude of the compression and shear waves are comparable but once combined, the total amplitude is reduced. This suggests that these two waves are similar in strength but out of phase. In the mid-frequency range $(1.5<\mathrm{St}<5)$, the shear wave signal is stronger than the compression wave, and the plateau in the combined spectrum is actually due to the shear wave. This confirms again that the dominant wave structure observed in Fig. 13 is generated by the shear wave mechanism. At higher frequencies ( $\mathrm{St}>10)$, the shear wave, which has a wave-length much smaller than the compression wave, dissipates more rapidly due to viscosity. Thus the compression wave is dominant in this higher frequency region, and the total spectrum in this range is almost identical to the one associated with the compression wave.

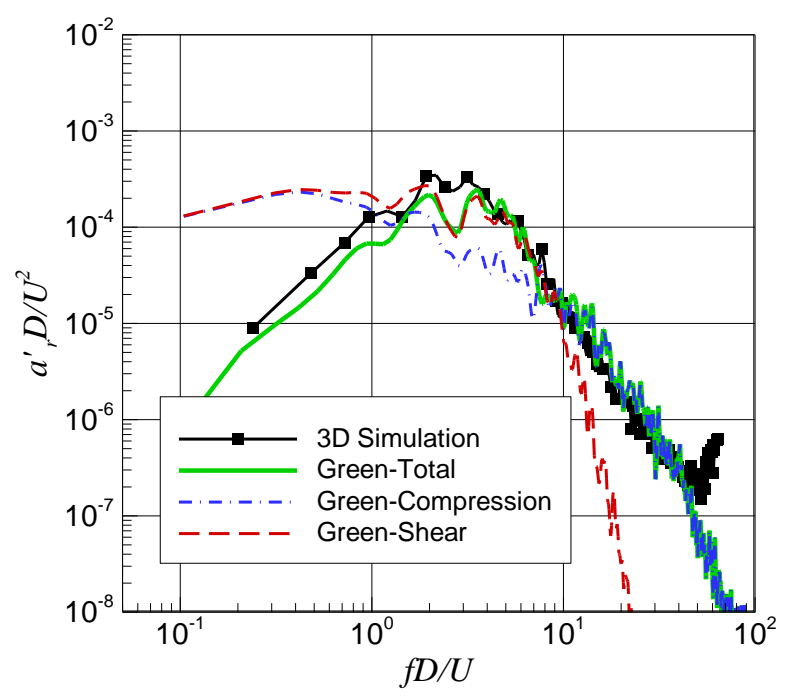

Figure 19: Frequency spectra of radial accelerations on the outer surface of tissue layer at the axial location of 2D downstream from the end of stenosis, 3D Simulation: computational hemoacoustic result, Green-Total: evaluated by using the free space Green's tensor, Green-Compression: result considering compression wave only, Green-Shear: shear wave only. 
The free space Green's tensor analysis therefore shows that both the compression and shear waves play important roles in the generation of the murmur signal, but the relative importance of each mechanism depends on the frequency range of interest. In the present case, the shear wave is responsible for the dominant wave structure at $1.5<\mathrm{St}<5$ (corresponding to 20 to $80 \mathrm{~Hz}$, approximately), but the effect of shear wave diminishes rapidly for higher frequencies due to the viscous damping, and the compression wave is dominant there.

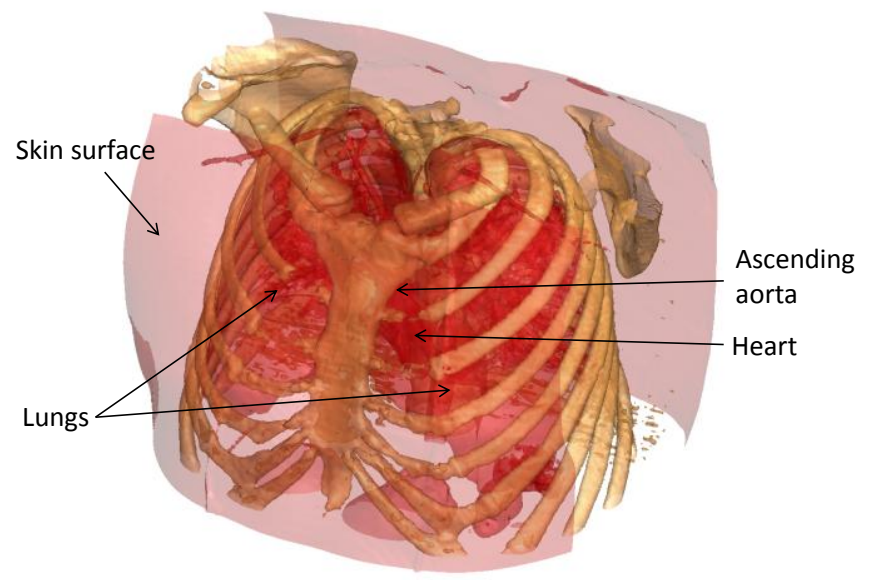

Figure 19: Real human thorax based on the CT scan data from the Visible Human Project.

\subsection{Murmur Propagation in Realistic Human Thorax}

As mentioned in the introduction, a real human thorax consists of many different biological materials and thus the waves associated with the murmur propagate through a complex, inhomogeneous medium. In order to examine the effect of this inhomogeneity, we simulate the propagation of the murmur in a realistic human thorax using the computational hemoacoustic method developed here. The realistic human thorax model is generated by using the CT (computed tomography) data from the Visible Human project ${ }^{\dagger}$ which is visualized in Fig. 19. The CT image data is processed and segmented to generate a surface grid for a geometry of the thorax (see Fig. 20A), and the different biological materials inside the thorax are modeled by choosing appropriate material properties. The density of the material is estimated based on the CT image contrast value using the formulation proposed in the previous study[54], and the compression and shear wave speeds are obtained based on Ref.[55]. The distributions of these material properties across one section of the thorax are shown in Fig. 20B.

\footnotetext{
${ }^{\dagger}$ An anatomical data set developed under a contract from the National Library of Medicine by the Departments of Cellular and Structural Biology, and Radiology, University of Colorado School of Medicine (https://www.nlm.nih.gov/research/visible/visible_human.html)
} 
For the source of murmur, we use the hemodynamic pressure fluctuation obtained from the flow simulation presented in Sec. 3.3.2. Particularly, the wall pressure fluctuation data at $z / D=2$ is used to synthesize the murmur source, and thus the source spectrum is the same as for the simpler model problem (Sec. 3.3). The murmur source is placed in the ascending aorta location inside the thorax model, and we used the form of Gaussian distributed pressure source term:

$$
S_{p, i j}=\delta_{i j} \frac{\partial P^{\prime}}{\partial t} \exp \left(\frac{\left(\vec{x}-\vec{x}_{A}\right)^{2}}{L_{p}^{2}}\right)
$$

where $P^{\prime}$ is the hemodynamic pressure fluctuation signal, $\vec{x}_{A}$ is the position vector of the ascending aorta location, and $L_{p}$ is the source length scale which is set to $1 \mathrm{~cm}$. The pressure source term, Eq. (34) is added to the right hand side of the first equation in Eq. (2). The thorax model is resolved by a uniform Cartesian grid with the resolution of $1 \mathrm{~mm}$, and grid with $300 \times 350 \times 250$ (about 26 million) points is used. The simulation for $0.8 \mathrm{sec}$ of physical time took about 201600 CPU hrs on the TACC-Stampede cluster. The murmur propagation is simulated by solving Eq. (2) using the present computational method. The traction free boundary condition is applied on the outer skin surface, and the non-reflecting, radiation boundary condition is applied at the $y$ and $z$ direction boundaries.

\section{A}

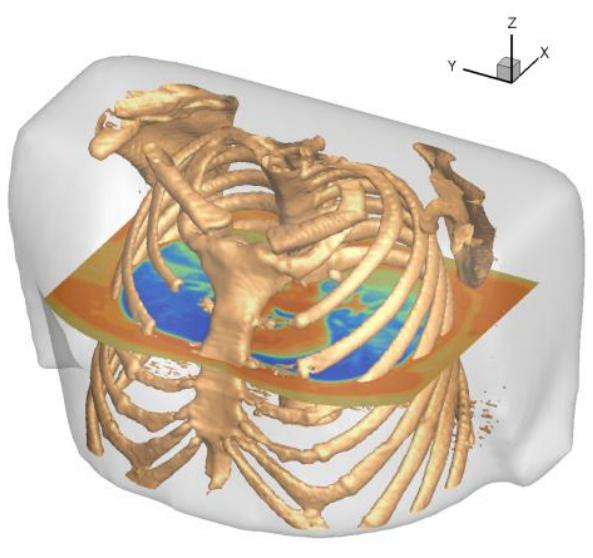



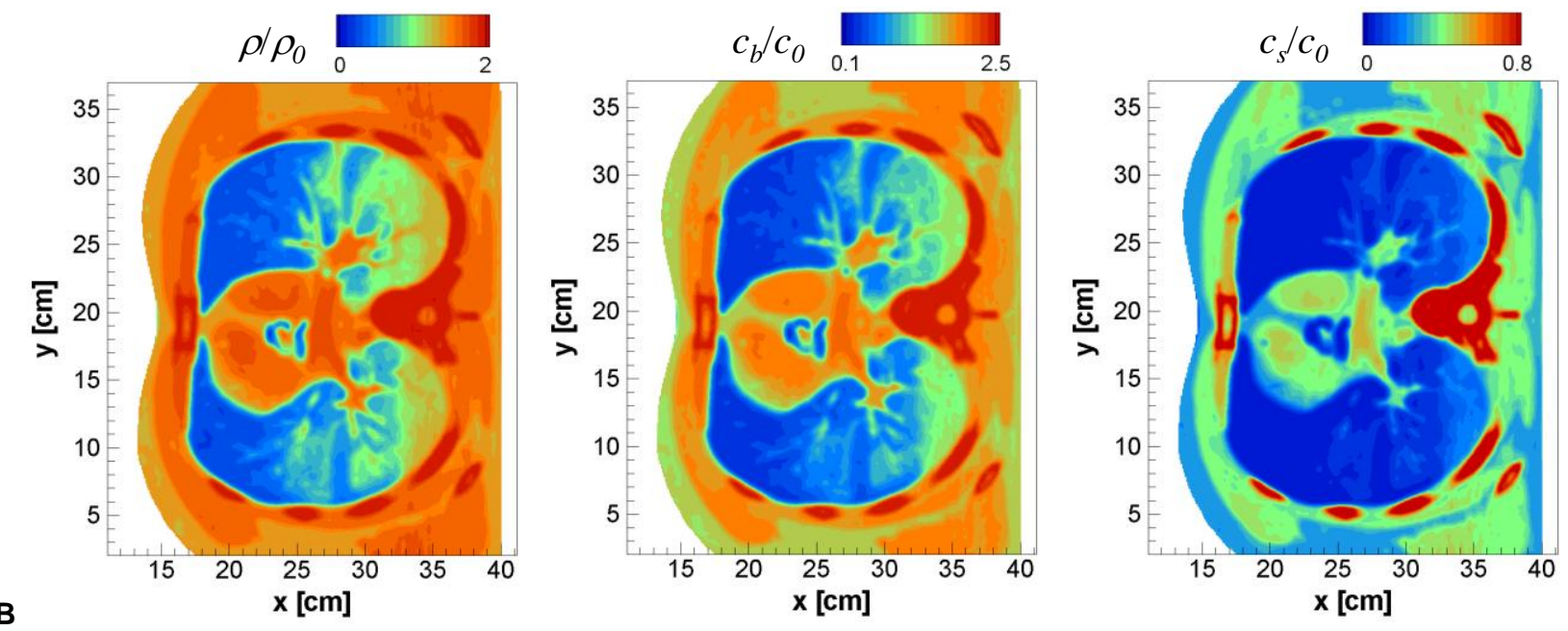

Figure 20: Realistic human thorax model generated using the CT scan data. A: Thorax geometry. The skin surface and the bones are visualized. B: Material property variations on the middle cross section. $\rho_{0}$ and $c_{0}$ are the density and speed of sound (compression wave) of the blood.
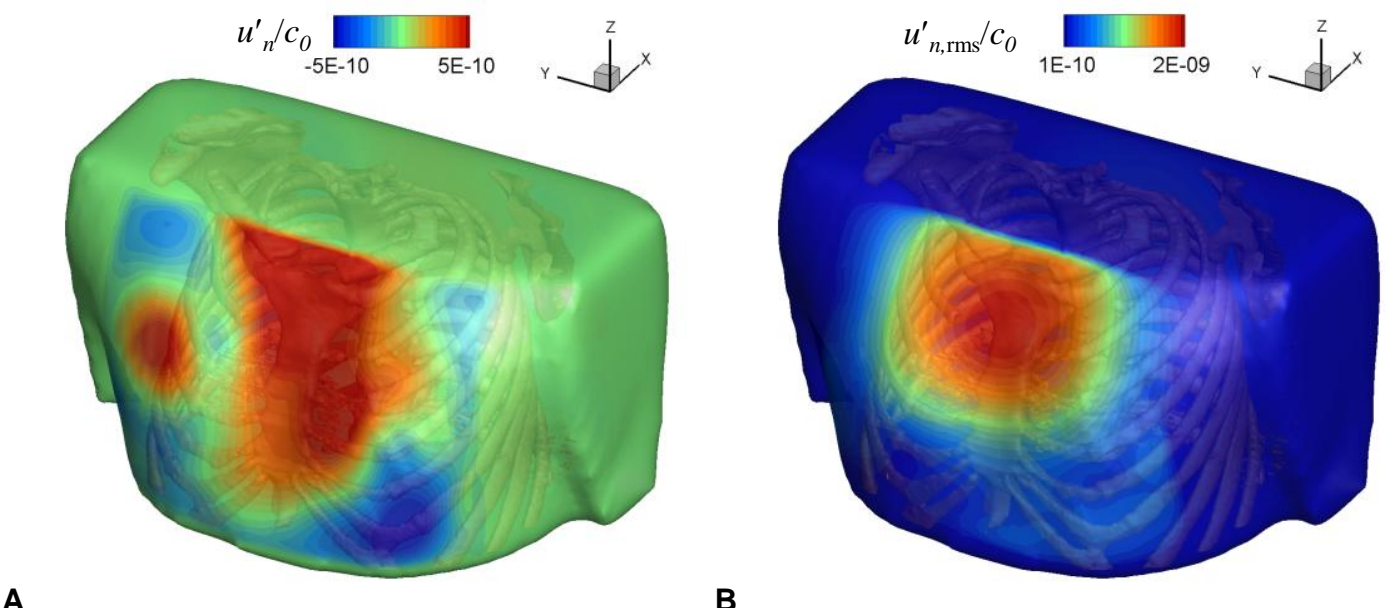

B

Figure 21: A: Instantaneous surface normal velocity fluctuation on the outer skin surface. B: Root-meansquared (rms) normal velocity fluctuation. 

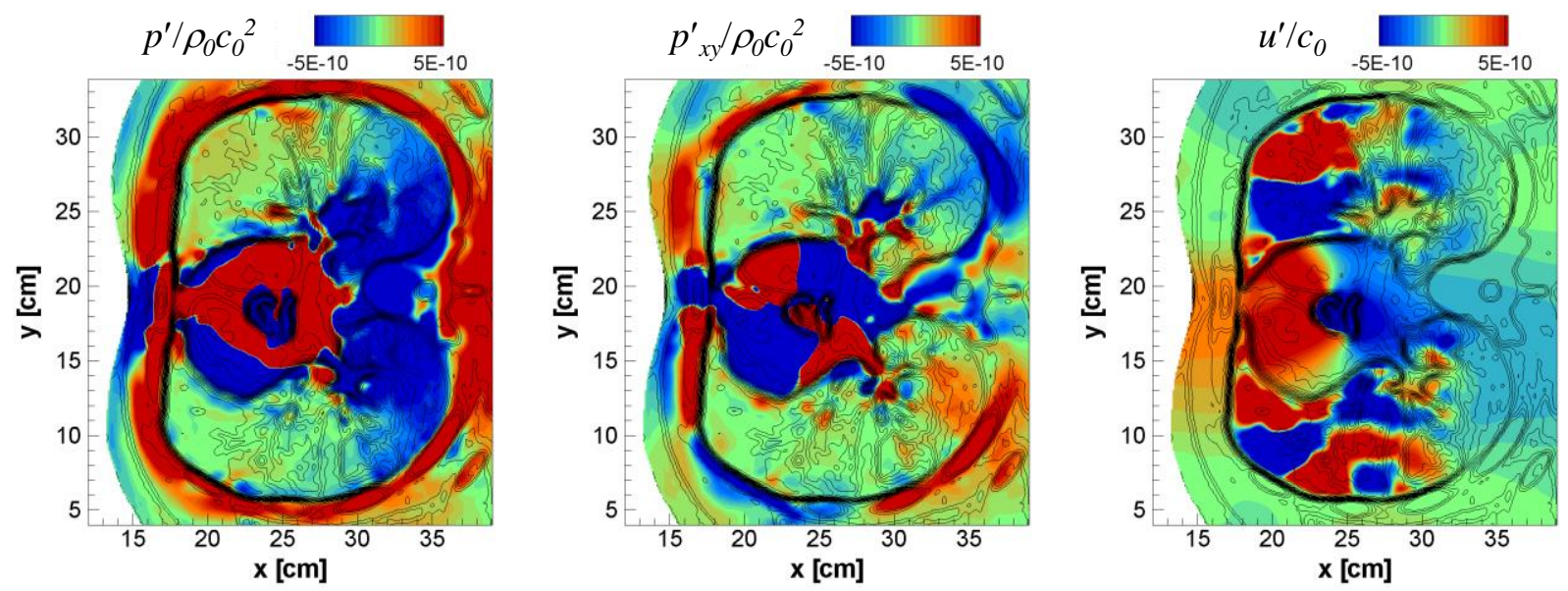

Figure 22: Instantaneous wave fields for the average normal stress $\left(p^{\prime}\right)$, shear stress $\left(p_{x y}^{\prime}\right)$, and $\mathrm{x}$ direction velocity fluctuation $\left(u^{\prime}\right)$. Black lines are the contours of the density.

The simulation results are presented in Figs. 21-23. Figure 21 shows the instantaneous and root-mean-squared (rms) surface normal velocity fluctuation on the outer skin surface. The strongest velocity fluctuation is observed around the surface directly above the source location (ascending aorta), but the instantaneous field also exhibits additional spatial variations on the surface. Instantaneous wave fields for the average normal stress $\left(p^{\prime}=\left(p_{x x}^{\prime}+p_{y y}^{\prime}+p_{z z}^{\prime}\right) / 3\right)$, shear stress $\left(p_{x y}^{\prime}\right)$, and velocity $\left(u^{\prime}\right)$ fluctuations are presented in Figure 22 . Due to low density and compression/shear modulus, the stresses in the lungs are quite weak. Interestingly, the velocity wave is also trapped in the lungs, but the wave generated in the heart muscle propagates through the gap between the lungs to the skin layer. The results indicate that the lungs are the primary obstacles for the murmur propagation from the heart to the outer skin surface. 


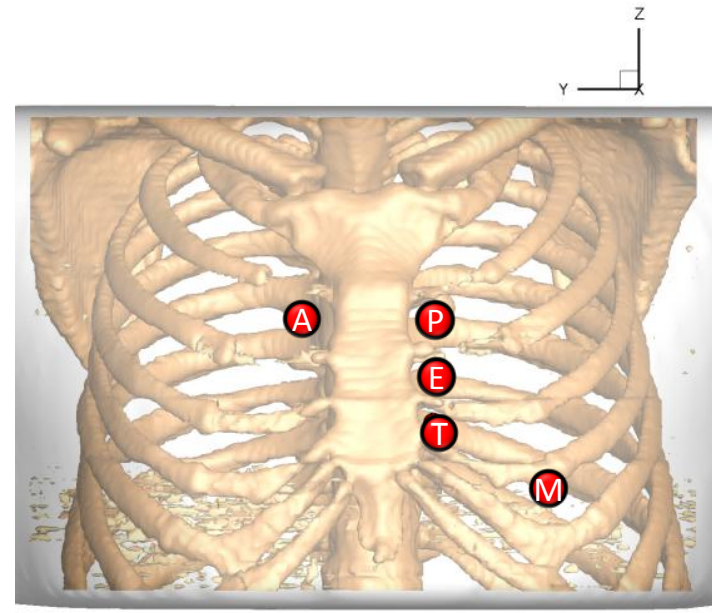

A

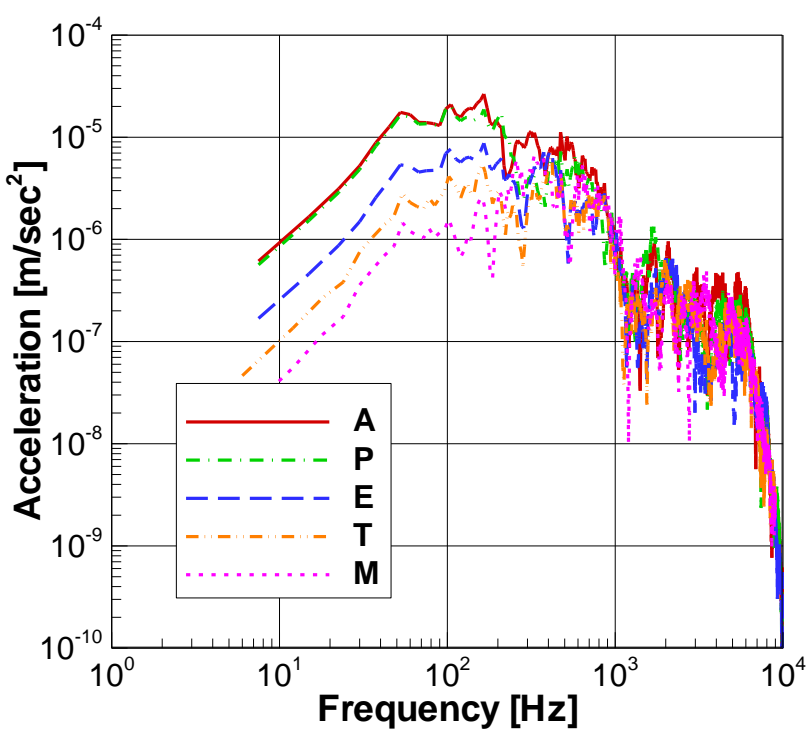

Figure 23: A: Traditional auscultation locations on the skin surface. (A) Aortic, (P) Pulmonic, (E) Erb's, (T) Tricuspid, and (M) Mitral point. B: Murmur spectrum at each auscultation point.

The propagated murmur signal (surface normal acceleration) is monitored at the five traditional auscultation points[56] which are marked in Fig. 23A and the frequency spectra of the monitored murmur at these five points are presented in Fig. 23B. The overall characteristics of the murmur spectrum are similar to what we have observed from the model problem (Sec. 3.3.3). The amplitude decreases for low frequencies $(<50 \mathrm{~Hz})$, a plateau region is observed for 50-200 Hz, and the energy decays for higher frequencies. Note that the actual frequency ranges for these three regimes are different from the model problem, because of the material properties of the medium. When compared the murmur spectrum of the model problem (Fig. 15), the realistic thorax results show more complex high frequency contents, which is clearly the effect of the inhomogeneity of the medium. Since the source of the murmur is in the ascending aorta, the signal monitored at the aortic point (A) shows the highest amplitude, and the amplitude decreases for the points farther from the aortic point. The spectrum at each location shows different frequency contents for the range $>200 \mathrm{~Hz}$, and this is also due to the effect of additional wave scattering by the inhomogeneous medium. The simulation results with the realistic thorax model suggest that even for the same source spectrum, the murmur spectrum can be modulated significantly by the thoracic organs and tissue.

By using these simulation results, it is straight-forward to construct transfer functions between the murmur source and the surface signals. Such transfer functions at the various surface locations can be used to develop a source 
localization technique involving the solution of an appropriate inverse problem. The present computational method will be used to build a simulation-based database of the transfer functions for the various murmur sources, which could be applied to advanced auscultation. This approach is currently being pursued and will be presented in the future.

\section{Concluding Remarks}

In the current study, we have proposed a computational "hemoacoustic" (CHA) method for the direct simulation of blood flow-induced sounds. The method is based on the immersed boundary framework to deal with complex geometries encountered in biological applications and employs high-order finite difference methods for the accurate resolution of wave propagation. The proposed method is verified against simple canonical problems with exact solutions and then applied to the model problem of an aortic stenosis murmur. The simulation results are validated by comparing with the experimental measurements of a companion experiment. Green's tensor analysis based on the computational results provides insights into the relative contribution of compression and shear waves on the detection of the murmur. This knowledge could potentially help in the development of new measurement techniques and improved sensors for more reliable auscultation. The present method is also applied to the simulation of murmur propagation in a relatively complex, realistic human thorax. These types of simulations could enable us to develop source localization methods based on the surface signals. In ongoing studies we are investigating the important effects of stenosis severity and flow pulsatility on the murmurs. Since the present method is capable of dealing with complex geometries, it is ready for application to more realistic heart murmur problems. Finally, the proposed immersed boundary, high-order simulation methods of elastic waves can also be applied for the accurate solution of the other body sounds such as intracranial or lung sounds.

\section{Acknowledgement}

This research is supported by NSF through grants IOS-1124804, IIS-1344772 and CBET-1511200. This work used the Extreme Science and Engineering Discovery Environment (XSEDE), which is supported by NSF grant number TG-CTS100002. 


\section{References}

[1] V.A. McKusick, Cardiovascular sound in health and disease, Am. J. Med. Sci.,238(1), pp:128 ,1959.

[2] D.L. Bruns, A general theory of the causes of murmurs in the cardiovascular system, Am. J. Med.,27(3), pp:360374,1959 .

[3] R.S. Lees and C.F. Dewey Jr, Phonoangiography: a new noninvasive diagnostic method for studying arterial disease, Proc. Natl. Acad. Sci. U. S. A.,67(2), pp:935-942,1970.

[4] P. Ask, B. Hök, D. Loyd and H. Teriö, Bio-acoustic signals from stenotic tube flow: state of the art and perspectives for future methodological development, Medical and Biological Engineering and Computing,33(5), pp:669-675, 1995 .

[5] W. Thompson, C. Hayek, C. Tuchinda, J. Telford and J. Lombardo, Automated cardiac auscultation for detection of pathologic heart murmurs, Pediatr. Cardiol.,22(5), pp:373-379 ,2001.

[6] I.R. Hanna and M.E. Silverman, A history of cardiac auscultation and some of its contributors, Am. J. Cardiol.,90(3), pp:259-267,2002.

[7] Y. Wen, A.P. Lee, F. Fang, C. Jin and C. Yu, Beyond auscultation: Acoustic cardiography in clinical practice, Int. J. Cardiol.,172(3), pp:548-560,2014.

[8] S.A. Ahmed and D.P. Giddens, Flow disturbance measurements through a constricted tube at moderate Reynolds numbers, J. Biomech.,16(12), pp:955-963 ,1983.

[9] J.J. Fredberg, Origin and character of vascular murmurs: Model studies, J. Acoust. Soc. Am.,61(4), pp:10771085,1977 .

[10] R.L. Kirkeeide, D.F. Young and N.R. Cholvin, Wall vibrations induced by flow through simulated stenoses in models and arteries, J. Biomech.,10(7), pp:431439-437441 ,1977.

[11] S.A. Jones and A. Fronek, Analysis of break frequencies downstream of a constriction in a cylindrical tube, J. Biomech.,20(3), pp:319-327,1987.

[12] R. Mittal, S. Simmons and F. Najjar, Numerical study of pulsatile flow in a constricted channel, J. Fluid Mech.,485 pp:337-378,2003.

[13] S.S. Varghese, S.H. Frankel and P.F. Fischer, Direct numerical simulation of stenotic flows. Part 1. Steady flow, J. Fluid Mech.,582 pp:253-280,2007.

[14] A. Borisyuk, Noise field in the human chest due to turbulent flow in a larger blood vessel, Flow, Turbulence and Combustion,61(1-4), pp:269-284,1998.

[15] J.J. Fredberg, Pseudo-sound generation at atherosclerotic constrictions in arteries, Bull. Math. Biol.,36(2), pp:143-155, 1974 .

[16] J.H. Seo and R. Mittal, A coupled flow-acoustic computational study of bruits from a modeled stenosed artery, Med. Biol. Eng. Comput.,50(10), pp:1025-1035,2012. 
[17] A.G. Andreou, T. Abraham, R. Thompson, J.H. Seo and R. Mittal, Mapping the cardiac acousteome: An overview of technologies, tools and methods, Information Sciences and Systems (CISS), 2015 49th Annual Conference on pp:1-6,2015.

[18] H. Bakhshaee, G. Garreau, G. Tognetti, K. Shoele, R. Carrero, T. Kilmar, C. Zhu, W.R. Thompson, J.H. Seo and R. Mittal, Mechanical design, instrumentation and measurements from a hemoacoustic cardiac phantom., Information Sciences and Systems (CISS), 2015 49th Annual Conference on pp:1-5 2015.

[19] T. Glozman and H. Azhari, A method for characterization of tissue elastic properties combining ultrasonic computed tomography with elastography, J. Ultrasound Med.,29(3), pp:387-398,2010.

[20] Y. Yazicioglu, T.J. Royston, T. Spohnholtz, B. Martin, F. Loth and H.S. Bassiouny, Acoustic radiation from a fluid-filled, subsurface vascular tube with internal turbulent flow due to a constriction, J. Acoust. Soc. Am.,118(2), pp:1193-1209,2005.

[21] M. Ozer, S. Acikgoz, T. Royston, H. Mansy and R. Sandler, Boundary element model for simulating sound propagation and source localization within the lungs, J. Acoust. Soc. Am.,122(1), pp:657-671,2007.

[22] T. Colonius and S.K. Lele, Computational aeroacoustics: progress on nonlinear problems of sound generation, Prog. Aerospace Sci.,40(6), pp:345-416,2004.

[23] C. Bailly, C. Bogey and O. Marsden, Progress in direct noise computation, Int. J. Aeroacoust,9(1-2), pp:123143,2010 .

[24] J.H. Seo, Y.J. Moon and B.R. Shin, Prediction of cavitating flow noise by direct numerical simulation, Journal of Computational Physics,227(13), pp:6511-6531,2008.

[25] J.H. Seo and S.K. Lele, Numerical investigation of cloud cavitation and cavitation noise on a hydrofoil section, 7th International Symposium on Cavitation CAV2009, Ann Arbor, USA,35,2009.

[26] R. Mittal and G. Iaccarino, Immersed boundary methods, Annu. Rev. Fluid Mech.,37 pp:239-261 ,2005.

[27] C.M. Otto and B. Prendergast, Aortic-valve stenosis - from patients at risk to severe valve obstruction, N. Engl. J. Med.,371(8), pp:744-756,2014.

[28] R.O. Bonow and P. Greenland, Population-wide trends in aortic stenosis incidence and outcomes, Circulation,131(11), pp:969-971,2015.

[29] A.J. Chorin, On the convergence of discrete approximations to the Navier-Stokes equations, Mathematics of Computation,23(106), pp:341-353,1969.

[30] R. Mittal, H. Dong, M. Bozkurttas, F. Najjar, A. Vargas and A. Von Loebbecke, A versatile sharp interface immersed boundary method for incompressible flows with complex boundaries, J. Comput. Phys.,227(10), pp:48254852,2008 .

[31] V. Vedula, S. Fortini, J. Seo, G. Querzoli and R. Mittal, Computational modeling and validation of intraventricular flow in a simple model of the left ventricle, Theor. Comput. Fluid Dyn.,28(6), pp:589-604,2014.

[32] H. Dong, M. Bozkurttas, R. Mittal, P. Madden and G. Lauder, Computational modelling and analysis of the hydrodynamics of a highly deformable fish pectoral fin, J. Fluid Mech.,645 pp:345-373,2010. 
[33] L. Zheng, T.L. Hedrick and R. Mittal, A multi-fidelity modelling approach for evaluation and optimization of wing stroke aerodynamics in flapping flight, J. Fluid Mech.,721 pp:118-154,2013.

[34] Y. Tanikaga, T. Sakaguchi and Y. Watanabe, A Study on analysis of intracranial acoustic wave propagation by the finite difference time domain method, Proceedings of Forum Acusticum, Sevilla,4(4.1), pp:4.2 ,2002.

[35] W.F. Hosford, Mechanical behavior of materials, Cambridge University Press, 2010.

[36] Y. Zheng, X. Chen, A. Yao, H. Lin, Y. Shen, Y. Zhu, M. Lu, T. Wang and S. Chen, Shear Wave Propagation in Soft Tissue with Ultrasound Vibrometry ,2013.

[37] A.I. Beltzer, Acoustics of solids, Springer Science \& Business Media, 2012.

[38] K.F. Graff, Wave motion in elastic solids, Courier Corporation, 1975.

[39] S.K. Lele, Compact finite difference schemes with spectral-like resolution, Journal of Computational Physics,103(1), pp:16-42,1992.

[40] A. Jameson, Numerical solution of the Euler equations for compressible inviscid fluids, in: Numerical Methods for the Euler Equations of Fluid Dynamics, F. Angrand, Institut National de Recherces en Informatique et Automatique. Workshop, ed., SIAM, Philadelphia, 1985.

[41] D.V. Gaitonde, J. Shang and J.L. Young, Practical aspects of higher-order numerical schemes for wave propagation phenomena, Int J Numer Methods Eng,45(12), pp:1849-1869,1999.

[42] H. Luo, R. Mittal, X. Zheng, S.A. Bielamowicz, R.J. Walsh and J.K. Hahn, An immersed-boundary method for flow-structure interaction in biological systems with application to phonation, Journal of computational physics,227(22), pp:9303-9332,2008.

[43] J.H. Seo and R. Mittal, A high-order immersed boundary method for acoustic wave scattering and low-Mach number flow-induced sound in complex geometries, Journal of computational physics,230(4), pp:1000-1019,2011.

[44] A. Ben-Menahem and S.J. Singh, Seismic waves and sources, Springer Science \& Business Media, 2012.

[45] N.B. Edgar and M.R. Visbal, A general buffer zone-type non-reflecting boundary condition for computational aeroacoustics, 9th AIAA/CEAS Aeroacoustics Conference and Exhibit, Aeroacoustics Conferences(AIAA 20033300), ,2003.

[46] T.J. Royston, Y. Yazicioglu and F. Loth, Surface response of a viscoelastic medium to subsurface acoustic sources with application to medical diagnosis, J. Acoust. Soc. Am.,113(2), pp:1109-1121,2003.

[47] A. Leatham, Systolic murmurs, Circulation,17(4), pp:601-611 ,1958.

[48] J.H. Seo and R. Mittal, Effect of diastolic flow patterns on the function of the left ventricle, Phys. Fluids,25(11), pp:0801,2013.

[49] T.S. Lund, X. Wu and K.D. Squires, Generation of turbulent inflow data for spatially-developing boundary layer simulations, Journal of Computational Physics,140(2), pp:233-258,1998.

[50] J. Jeong and F. Hussain, On the identification of a vortex, J. Fluid Mech.,285 pp:69-94,1995. 
[51] D. Einstein, K. Kunzelman, P. Reinhall, M. Nicosia and R. Cochran, Haemodynamic determinants of the mitral valve closure sound: a finite element study, Medical and Biological Engineering and Computing,42(6), pp:832846,2004 .

[52] M. Wang, J.B. Freund and S.K. Lele, Computational prediction of flow-generated sound, Annu. Rev. Fluid Mech.,38 pp:483-512,2006.

[53] M. Wang and P. Moin, Computation of trailing-edge flow and noise using large-eddy simulation, AIAA J.,38(12), pp:2201-2209,2000.

[54] C. Narasimhan, R. Ward, K.L. Kruse, M. Guddati and G. Mahinthakumar, A high resolution computer model for sound propagation in the human thorax based on the Visible Human data set, Comput. Biol. Med.,34(2), pp:177192,2004 .

[55] S. Ramakrishnan, S. Udpa and L. Udpa, A numerical model simulating sound propagation in human thorax, Biomedical Imaging: From Nano to Macro, 2009. ISBI '09. IEEE International Symposium on pp:530-533,2009.

[56] J.S. Coviello, Auscultation Skills: Breath \& Heart Sounds, Lippincott Williams \& Wilkins, 2013. 